OPEN ACCESS

Edited by:

Pan Zheng,

University of Canterbury, New Zealand

Reviewed by:

Enqiang Zhu,

Guangzhou University, China

Xuncai Zhang,

Zhengzhou University of Light

Industry, China

*Correspondence:

Changjun Zhou

zhou-chang231@163.com

Specialty section:

This article was submitted to

Computational Genomics,

a section of the journal

Frontiers in Genetics

Received: 17 January 2021

Accepted: 09 February 2021

Published: 04 March 2021

Citation:

Xue XL, Jin HY, Zhou DS and

Zhou CJ (2021) Medical Image

Protection Algorithm Based on

Deoxyribonucleic Acid Chain

of Dynamic Length.

Front. Genet. 12:654663.

doi: 10.3389/fgene.2021.654663

\section{Medical Image Protection Algorithm Based on Deoxyribonucleic Acid Chain of Dynamic Length}

\author{
Xianglian Xue 1,2, Haiyan Jin ${ }^{1,3}$, Dongsheng Zhou ${ }^{4}$ and Changjun Zhou ${ }^{5 *}$ \\ ${ }^{1}$ School of Computer Science and Engineering, Xi'an University of Technology, Xi'an, China, ${ }^{2}$ Sections of Computer \\ Teaching and Research, Shaanxi University of Chinese Medicine, Xianyang, China, ${ }^{3}$ Shaanxi Key Laboratory for Network \\ Computing and Security Technology, Xi'an University of Technology, Xi'an, China, ${ }^{4}$ Key Laboratory of Advanced Design \\ and Intelligent Computing, Ministry of Education, Dalian University, Dalian, China, ${ }^{5}$ College of Mathematics and Computer \\ Science, Zhejiang Normal University, Jinhua, China
}

Current image encryption algorithms have various deficiencies in effectively protecting medical images with large storage capacity and high pixel correlation. This article proposed a new image protection algorithm based on the deoxyribonucleic acid chain of dynamic length, which achieved image encryption by DNA dynamic coding, generation of DNA dynamic chain, and dynamic operation of row chain and column chain. First, the original image is encoded dynamically according to the binary bit from a pixel, and the DNA sequence matrix is scrambled. Second, DNA sequence matrices are dynamically segmented into DNA chains of different lengths. After that, row and column deletion operation and transposition operation of DNA dynamic chain are carried out, respectively, which made DNA chain matrix double shuffle. Finally, the encrypted image is got after recombining DNA chains of different lengths. The proposed algorithm was tested on a list of medical images. Results showed that the proposed algorithm showed excellent security performance, and it is immune to noise attack, occlusion attack, and all common cryptographic attacks.

\footnotetext{
Keywords: FOCHC system, DNA dynamic encoding, DNA dynamic chain, medical image encryption, deletion and transposition operation
}

\section{INTRODUCTION}

Nowadays, technologies such as telemedicine, tele-surgery, and tele-radiology have been enormously developed and are in the preparation stage for clinical usage (Priyanka and Maheshkar, 2017). Patient information may be exposed to network transmission with these technologies. Especially, medical images (MRI, CT, and X-ray) with large data storage, redundancy and high pixel correlation are easily attacked and tampered by unauthorized access. Therefore, it is necessary to develop efficient high-performance medical image encryption method.

Since the ground-breaking work on DNA computing conducted and reported by Adleman (1994). DNA computing has attracted ever increasing attention of researchers worldwide, due to its superior characteristics of large concurrency, mass storage and low energy consumption (Li et al., 2020; Liu et al., 2020; Wang B. et al., 2020; Zhu et al., 2020). In 2009, DNA coding theory was used in the field of image information security by Zhang et al. (Xue et al., 2010a,b; Zhang et al., 2010; Liu et al., 2012; Zhang and Wei, 2013), which opened a new window for the DNA cryptography. 
The main encryption ideas were using the DNA operations (addition, subtraction, XOR, and DNA complement operations) and combination with some chaotic systems to achieve image encryption. Their novel methods and better encryption effects were often emulated and affirmed by researchers. However, Zhang et al.'s method was criticized as being unsafe in recent years. For instance, Zhu et al. (2017) and Hermassi et al. (2014) pointed out that the DNA addition operation proposed by Zhang et al. (2010) was irreversible. Besides, the encryption algorithm proposed by Zhang et al. (2012) has been deciphered by Belazi et al. (2014), Liu et al. (2014), and Wang et al. (2015) with chosen plaintext attack (CPA), respectively.

To improve the security, some researchers combined the complex chaotic systems with the DNA coding. For instance, Mondal and Mandal (2017) used two Logistic chaotic systems, and Zhang Y.Q. et al. (2016) used MLNCML system embedded logistic, to strengthen the existing algorithm. All of them combined the chaotic system with the DNA coding operations (addition and subtraction) to encrypt images. Zhang and Gao (2016) proposed an image encryption method which used hyperchaotic system to control the DNA complement operation. However, because they adopted the technique of fixed DNA coding and fixed operation rules, the security of their algorithm was quite fragile. Further, the encryption key had not been associated with the original image. As a result, although complex chaotic systems was used to improve the security of the algorithm, the encrypted images could still be easily deciphered by the CPA and brute force attack (BFA) or the known plaintext attack (KPA) (Dou et al., 2017). Note that chaotic systems play a major role in such encryption methods, while the DNA coding operation without chaotic mapping was equivalent to the calculation of binary bits, and its security was not guaranteed. For example, Kumar et al. (2016) proposed a technique using DNA coding combined with elliptic curve Diffie-Hellman for image encryption, while it was deciphered by Akhavan et al. (2017) at no much cost using the chosen plaintext attack.

For these reasons, researchers used more efficient DNA coding mechanisms and DNA operations to achieve better performance chaotic systems for image encryption. In terms of DNA coding, Kalpana and Murali (2015), Zhen et al. (2016), Chai et al. (2017), Rehman et al. (2018), Dagadu et al. (2019a), and Hossein et al. (2020), etc. proposed different DNA dynamic coding, respectively. These methods gave DNA bases higher levels of encryption. However, all of the above dynamic DNA coding were based on image blocks or based on pixel-by-pixel. In addition, some of them (Dagadu et al., 2019a; Hossein et al., 2020) could not resist CPA. In terms of the DNA operations, dynamic addition operation (Zhang J. et al., 2016) and complement operation (Belazi et al., 2019), and cellular automata operation (Zhou et al., 2016; Chai et al., 2017) were proposed. Their encryption effects were better but the algorithms were more complex. In terms of the chaotic system, because the hyper-chaotic system obtained by fractional order calculation had low correlation and more complex dynamic characteristics (Zhu et al., 2014), it was favored by researchers. For example, Zhang L.M. et al. (2017) used the fractional-order hyper-chaotic system (FOHC) to scramble the DNA sequence, and achieved better image encryption effect. Li et al. (2017) used the fractionalorder Lorenz hyper chaotic mapping (FOLHC) to direct the DNA operations (XOR, addition, subtraction). However, these methods were complex and the keys used were independent of the original images.

In this article, a medical image protection method based on dynamic deoxyribonucleic acid chain operation is proposed. The algorithm is tested against three kinds of medical images, and the performance, safety, efficiency of the developed algorithm evaluated against existing algorithms reported in the literature. The general arrangement for this article is as follows: First, DNA dynamic coding, FOCHC, and DNA chain operation are introduced in section "Background Knowledge." Then, section "The Proposed Algorithm" introduces the proposed method. Next, section "Simulation Results" simulates the results. Security analyses are shown in the section "Security Analyses" and the conclusion in the section "Conclusion."

\section{BACKGROUND KNOWLEDGE}

\section{DNA Coding Rule}

There are four bases in a deoxyribonucleic acid chain. They are adenine $(A)$, cytosine $(C)$, guanine $(G)$, thymine $(T)$, in which $A$ and $T$ complement with each other, so do $C$ and $G$. The four bases are denoted by the binary numbers of $00,01,10$, and 11 , normally. A total of 24 types of coding have been list (Xue et al., 2010b). However, because 0 and 1 complement with each other in binary, so do 01 and 10, and 00 and 11 . Thus, only 8 of the 24 DNA coding rules satisfy the principle of base complementary, as shown in Table 1.

By summarizing and categorizing the existing dynamic coding cases, it is observed that the existing cases fall into three categories: (1) those are based on the image block (column/row) dynamic coding (Akhavan et al., 2017); (2) those are based on the pixel dynamic encoding (Kalpana and Murali, 2015; Dagadu et al., 2019b; Wang X.Y. et al., 2020); and (3) those are based on the binary bit dynamic coding (Zhang J. et al., 2016). Because a DNA chain contains four bases, theoretically each base should appear in a random image with $25 \%$ of probability. The following equation can be used to calculate the base distribution rate of the above different DNA dynamic coding. The result is shown in Table 2.

$$
A P=\operatorname{count}(A) \div(M \times N \times 4) \times 100 \%
$$

\begin{tabular}{lcccccccc}
\hline \multicolumn{2}{l}{ TABLE 1 } & Eight kinds of DNA coding rules. \\
\hline Binary & R1 & R2 & R3 & R4 & R5 & R6 & R7 & R8 \\
\hline 00 & A & A & C & C & G & G & T & T \\
01 & C & G & A & T & A & T & C & G \\
10 & G & C & T & A & T & A & G & C \\
11 & T & T & G & G & C & C & A & A \\
\hline
\end{tabular}


TABLE 2 | The base distribution rate of different kinds of dynamic coding.

\begin{tabular}{lccc}
\hline Base distribution & By row (\%) & By pixel (\%) & By bit (proposed) (\%) \\
\hline AP & 24.50 & 24.90 & 24.98 \\
TP & 24.65 & 24.90 & 25.04 \\
CP & 25.42 & 25.33 & 24.97 \\
GP & 25.43 & 24.87 & 25.01 \\
\hline
\end{tabular}

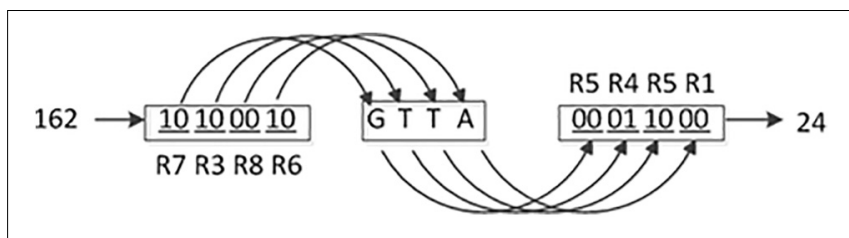

FIGURE 1 | The process diagram of DNA dynamic encoding and decoding by binary bit.

This equation uses base " $\mathrm{A}$ " as the example, where $\mathrm{M}$ and $\mathrm{N}$ are the numbers of row and column in the image; count (A) is the counting function of base "A." A pixel consists of eight bits of binary, so four bases can represent one pixel. Here $\mathrm{M} \times \mathrm{N} \times 4$ is the total number of the possible base appearance. The distribution rate of "T," "C," and " $\mathrm{G}$ " can be calculated similarly.

From Table 2, it is found that the values of the base distribution rate of the DNA dynamic coding by binary bit are close to $25 \%$, and the maximum deviation of the base distribution rates from $25 \%$ is $0.04 \%$. Consequently, the DNA dynamic coding by binary bit is used for encoding and decoding in this study. A detailed coding example is shown in Figure 1. Where (R7, R3, R8, and R6) and (R5, R4, R5, and R1) are the encoding and decoding rules from Table 1, respectively, and they are controlled by the chaotic map. It can be seen that the image pixel value changes from 162 to 24. To our knowledge, this is the best DNA coding for image encryption.

\section{Fractional-Order Chen Hyper Chaotic (FOCHC) System}

It is well known that hyper chaotic systems have much advantage over low-dimensional chaotic systems or multi-chaotic combination systems. Also the fractional-order hyper chaotic systems are superior to integer-order hyper chaotic systems in several aspects, including cross-correlation, self-correlation amplitude, pseudo-randomness, and the correlation and so on (Zhu et al., 2014).

Among the common fractional-order decomposition algorithms to solve the fractional-order chaotic system, the Adomian decomposition algorithm is the best choice since it has high precision, low complexity, and high computational efficiency (Donato and Giuseppe, 2008). Thus the Adomian decomposition algorithm is chosen to solve the FOCHC in this study, and the generated chaotic sequence is then used for image encryption. The FOCHC system model is described below:

$$
\left\{\begin{array}{c}
\frac{d^{q}}{d t^{q}} x=a(y-x)+w \\
\frac{d^{q}}{d t^{q}} y=b x-x z+c y \\
\frac{d^{q}}{d t^{q}} z=x y-d z \\
\frac{d^{q}}{d t^{q}} w=y z-e w
\end{array}\right.
$$

When $\mathrm{a}=38, \mathrm{~b}=7, \mathrm{c}=12, \mathrm{~d}=3$, e $=0.7$, the system is in chaotic state and four chaotic sequences $\mathrm{x}, \mathrm{y}, \mathrm{z}, \mathrm{w}$ are generated. The chaotic attractors for $q=0.98$ are shown in Figure 2 .

\section{The Definition of DNA Chain Operation}

The DNA chain is defined as:

$$
C_{n}=C_{h} C_{h-1} \ldots C_{2} C_{1}(h \leq n)
$$

Here, $C_{n}$ is a DNA chain with length $\mathrm{m}$. It can be broken into smaller DNA chains of $C_{h}, C_{h-1}, \ldots C_{2}, C_{1}$, with different lengths of $L_{h}, L_{h-1} \ldots L_{2}, L_{1}$, respectively. Apparently, $m=L_{h},+L_{h-1}+\ldots L_{2}+L_{1}$. In the DNA computing, there are several operations on the DNA chain to achieve base scrambling, including the deletion, the insertion, and the transposition operations. Their operating principles are shown in Figure 3.

\section{THE PROPOSED ALGORITHM}

The proposed new method in this study includes the following four steps. Firstly, the original image is encoded into a DNA matrix dynamically, by using a FOCHC sequence. Secondly, the DNA matrix is scrambled by two other FOCHC sequences. Thirdly, DNA dynamical chain operations are carried out by four FOCHC sequences. At last, the DNA matrix generated is decoded into a binary matrix by a FOCHC sequences, and the encrypted image is obtained after recombining the DNA chain. Eight chaotic sequences are used to complete the above four steps. The eight sequences are generated by two FOCHC under different keys, which are obtained using the SHA-256 algorithm and the hamming distance. The detailed steps and the flowchart are shown in section "Key Generation," section "Key Generation by SHA-256," section "Key Generation by Hamming Distance, Generation of FOCHC sequences," section "Scrambling of the DNA sequence matrix," section "The proposed algorithm Based on the DNA dynamic chains operation," section "Generation of the dynamic DNA chains," section "Deletion operation on the dynamic DNA chain," section "Transposition operation on the dynamic DNA chain," section "Insertion operation on the dynamic DNA chain," and section "The proposed algorithm" and Figure 4.

\section{Key Generation}

Two kinds of keys are used as the initial values of the FOCHC sequences, and they are generated by the SHA-256 algorithm 


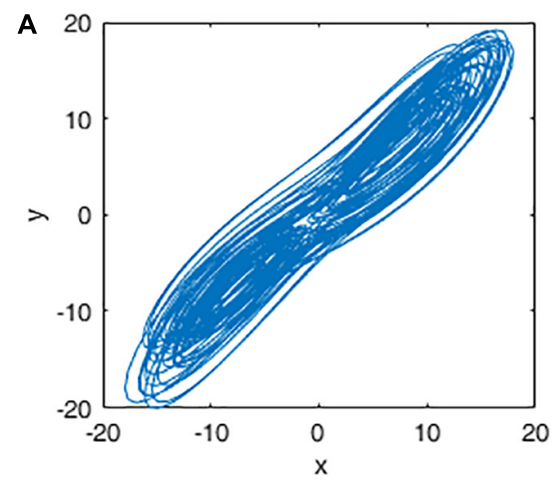

C

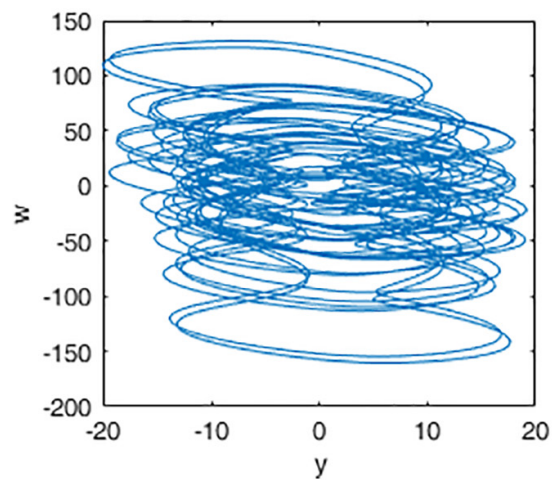

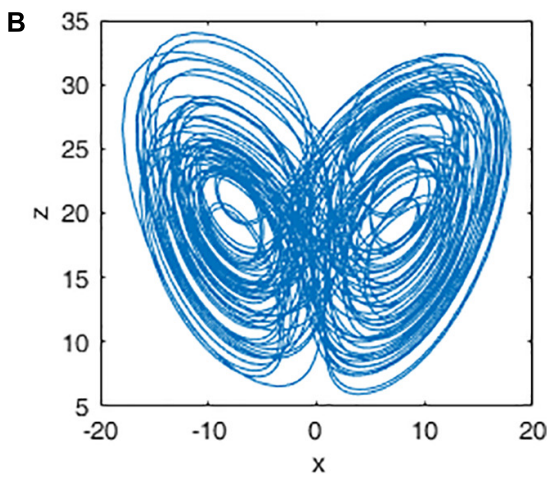

D

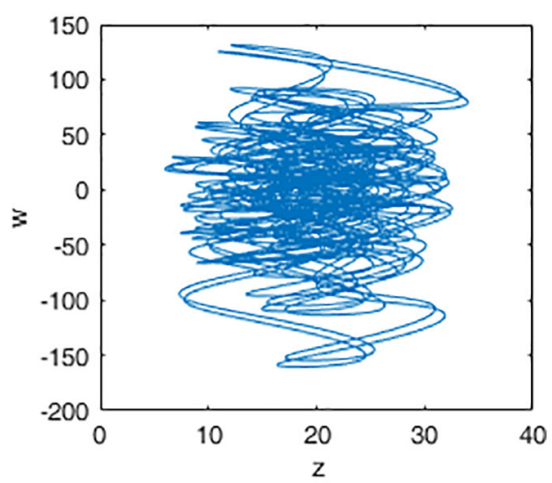

FIGURE 2 | Attractors of the fractional-order Chen hyper chaotic system: (A) x-y plane; (B) x-z plane; (C) y-w plane; and (D) z-w plane.

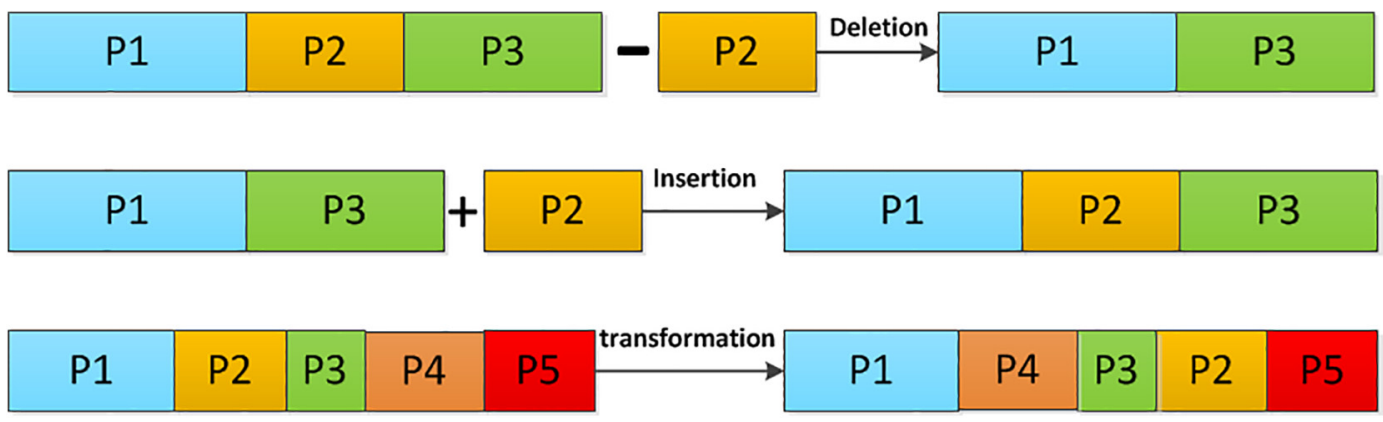

FIGURE 3 | The operating principle of DNA chains.

and the hamming distance, respectively. The former effectively defends against the KPA and CPA, and the latter enhances the diffusion ability of the bases.

\section{Key Generation by SHA-256}

The key generation in this algorithm depends on the SHA-256 function proposed in Belazi et al. (2019), because the result of the SHA-256 function is sensitive to the original image changes, since even one pixel change can result in a completely different hash value. In this study the 256-bit hash value is obtained by applying the SHA-256 function to the original image at first. The value is then converted into decimal numbers in groups of eight bits, and a decimal sequence $\mathrm{K}$ of length 32 is obtained, which can be expressed as $\mathrm{K}=\{\mathrm{K} 1, \mathrm{~K} 2 \ldots \mathrm{K} 32\}$. The initial values are obtained via $\mathrm{K}$; the detail equation is defined below. 


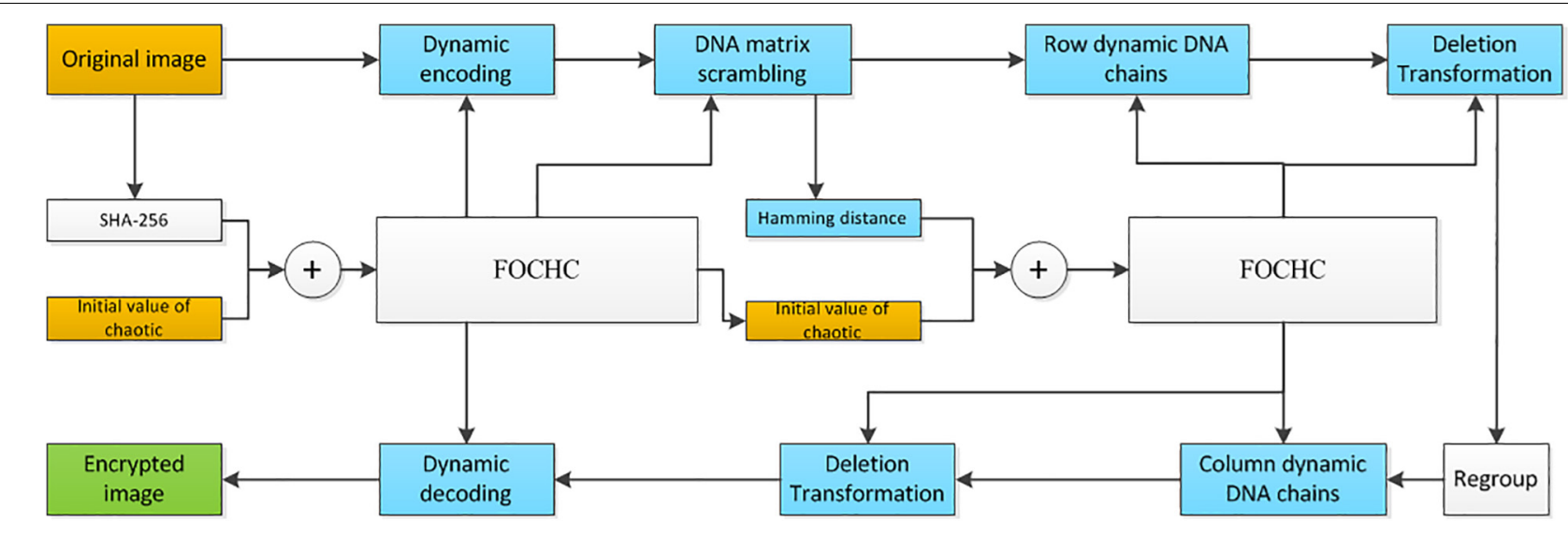

FIGURE 4 | The flowchart of the proposed algorithm: yellow blocks are the input, green block is the output, and blue blocks from the DNA coding algorithm.

$$
\left\{\begin{array}{l}
x \_s=k 1+x 0 \\
y \_s=k 2+y 0 \\
z \_s=k 3+z 0 \\
w \_s=k 4+w 0
\end{array}\right.
$$

Where $x \_s, y \_s, z \_s, w \_s$ are the initial values obtained, and $\mathrm{x} 0$, $\mathrm{y} 0, \mathrm{z} 0$, and $\mathrm{w} 0$ are the initial values given.

\section{Key Generation by Hamming Distance}

There are four steps for key generation by the hamming distance, as detailed in the following:

Step 1: For a DNA matrix A_DNA_maxtrix $(m, n \times 4)$, calculate the hamming distance for every two rows and every two columns of the matrix, respectively. The row hamming distance $\mathrm{R}_{\mathrm{H}}=\left\{\mathrm{r}_{\mathrm{h} 1}, \mathrm{r}_{\mathrm{h} 2} \ldots, \mathrm{r}_{\mathrm{hi}}, \ldots \mathrm{r}_{\mathrm{hm} / 2}\right\}$ and the column hamming distance $\mathrm{C}_{\mathrm{H}}=\left\{\mathrm{c}_{\mathrm{h} 1}, \mathrm{c}_{\mathrm{h} 2} \ldots, \mathrm{c}_{\mathrm{hi}} \ldots \mathrm{c}_{\mathrm{hn}=4 / 2}\right\}$ are obtained. The equation for calculating the hamming distance is:

$$
\left\{\begin{aligned}
D(M, N) & =\sum_{i=0}^{L} d\left(m^{\prime}{ }_{i}, n^{\prime}{ }_{i}\right) \\
d\left(m_{i,}^{\prime} n_{i}^{\prime}\right) & =\left\{\begin{array}{l}
0, \text { if } m^{\prime}{ }_{i}=n^{\prime}{ }_{i} \\
1, \text { if } m^{\prime}{ }_{i} \neq n^{\prime}{ }_{i}
\end{array}\right.
\end{aligned}\right.
$$

Where $m_{\mathrm{i}}^{\prime}$ and $n^{\prime}{ }_{\mathrm{i}}$ are the ith base of the DNA chains M and $\mathrm{N}$, respectively, and $\mathrm{D}(\mathrm{M}, \mathrm{N})$ is the hamming distance between $\mathrm{M}$ and $\mathrm{N}$;

Step 2: Calculate the average values of $\mathrm{R}_{\mathrm{H}}$ and $\mathrm{C}_{\mathrm{H}}$, which are $\mathrm{R}_{\mathrm{h}}^{\prime}$ and $\mathrm{C}_{\mathrm{h}}^{\prime}$, respectively.

Step 3: Extract the decimal parts $\mathrm{p}$ and $\mathrm{q}$ from $\mathrm{R}_{\mathrm{h}}^{\prime}$ and $\mathrm{C}_{\mathrm{h}}^{\prime}$. Step 4: The new initial values of FOCHC are obtained by Eq. (6). $\mathrm{x}^{\prime}, \mathrm{y}^{\prime}, \mathrm{z}^{\prime}$, and $\mathrm{w}^{\prime}$ are the given initial values, $\mathrm{k} 5, \mathrm{k} 6, \mathrm{k} 7, \mathrm{k} 8$ are calculated as described in section "Key Generation by SHA-256."

$$
\left\{\begin{aligned}
X_{h} & =k 5+p+x 0^{\prime} \\
Y_{h} & =k 6+p+y 0^{\prime} \\
Z_{h} & =k 7+q+z 0^{\prime} \\
W_{h} & =k 8+q+w 0^{\prime}
\end{aligned}\right.
$$

\section{Generation of FOCHC Sequences}

The initial values generated using the FOCHC sequences, as described in section "Key generation," was input into the FOCHC system to produce four groups of chaotic sequences $\mathrm{X}, \mathrm{Y}, \mathrm{Z}, \mathrm{W}$ after being iterated for $1000+\mathrm{m} \times \mathrm{n} \times 4$ times. To eliminate the transient effects in the chaotic systems, the chaotic sequences were recalculated for 1,000 iterations before being used, and their length were $\mathrm{m} \times \mathrm{n} \times 4$.

\section{Scrambling of the DNA Sequence Matrix}

Step 1: Input the DNA sequence matrix $A(m, n \times 4)$, whose size is $(\mathrm{m}, \mathrm{n} \times 4)$;

Step 2: Use the following equations to transform the chaotic sequences:

$$
\left\{\begin{array}{l}
Y Y=a b s(Y 1-f i x(Y 1)) \\
Z Z=a b s(Z 1-f i x(Z 1))
\end{array}\right.
$$

Where, $\mathrm{Y} 1$ and $\mathrm{Z} 1$ are the FOCHC sequences. The length of $\mathrm{Y} 1$ is $\mathrm{m}$, and the length of $\mathrm{Z} 1$ is $\mathrm{n} \times 4$. fix(.) is the rounding function. $\operatorname{abs}($.$) is the absolute value function.$

Step 3: Sort YY and ZZ, respectively, to obtain the index values By and Bz.

$$
\left\{\begin{array}{l}
{[\sim, B y]=\operatorname{sort}(Y Y)} \\
{[\sim, B z]=\operatorname{sort}(Z Z)}
\end{array}\right.
$$

Step 4: Use the following equation to scramble A, and obtain the matrix A_scrambing.

$$
A \_\operatorname{scrambing}(i, j)=A(B y(i), B z(j)) ;
$$

Where $\mathrm{i}=1,2 \ldots \mathrm{m}, \mathrm{j}=1,2 \ldots \mathrm{n} \times 4$.

\section{The Proposed Algorithm Based on the DNA Dynamic Chains Operation}

Through DNA dynamic chain operation, the algorithm proposed changes the position of the base, which leads to changes of the pixel values in the image. As shown in Figure 5, each row in the DNA sequence matrix is divided into chains of 


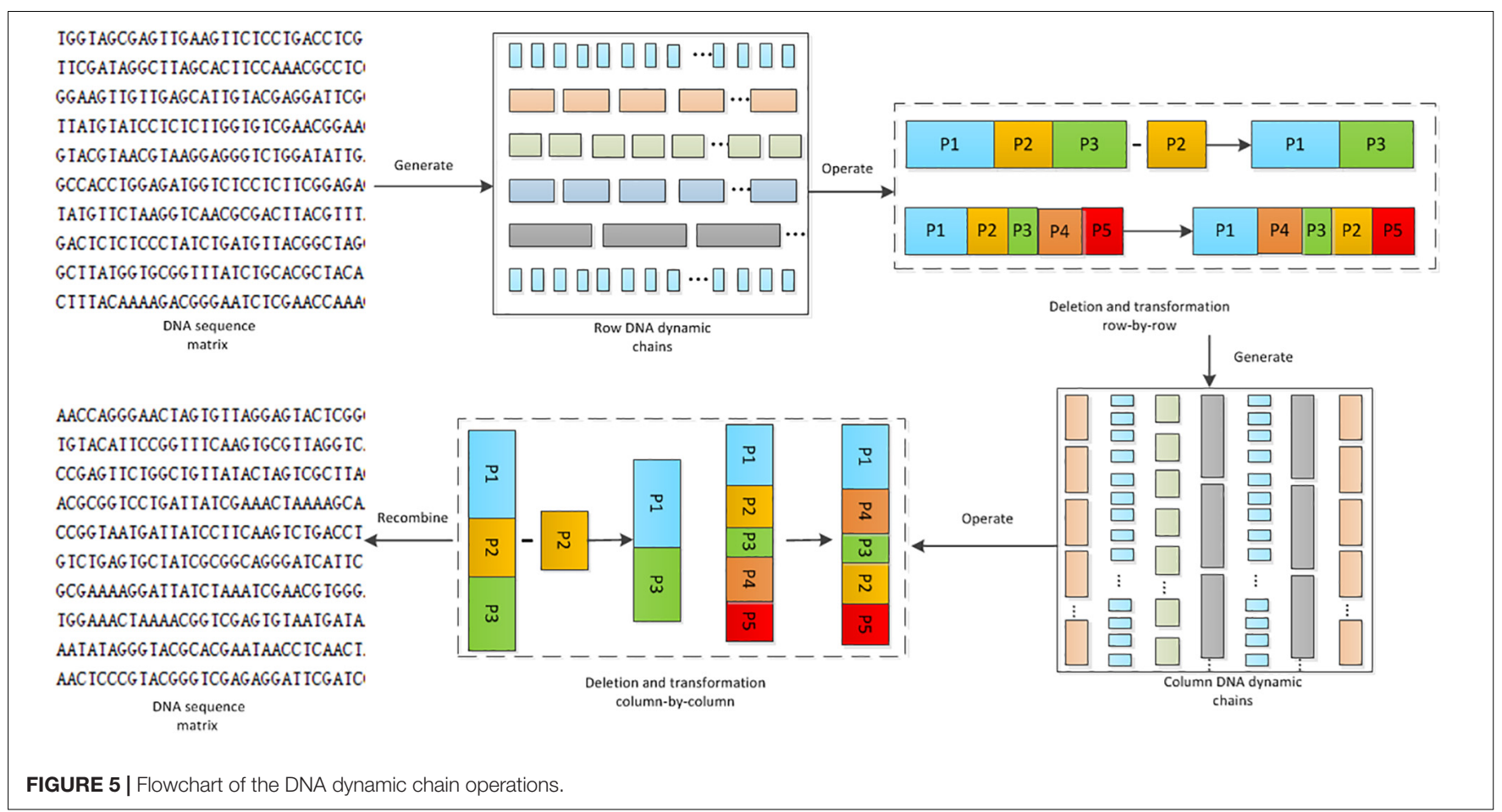

different lengths. Deletion operation and transposition operation are applied on these chains. This is the first shuffle process on the DNA sequence matrices. To eliminate the block effect, generation of the column DNA dynamic chain, and the deletion and transposition operations are conducted again. Thus the DNA sequence matrices are scrambled for the second time. In the whole process, the length of the DNA chain is dynamic, so is the operation, and the DNA base is completely disrupted.

\section{Generation of the Dynamic DNA Chains}

DNA sequence matrix is divided into DNA chains of different lengths by rows or columns. The length of the chain is controlled by the hyper-chaotic sequence. The following explains the detailed steps for generating DNA dynamic chains row-by-row. Generation of the DNA dynamic chains column-by-column can be implemented in a similar way, except that the DNA matrix needs to be transposed before the generation.

Step 1: A FOCHC sequence $\mathrm{x} 1$ is transformed using the following equation:

$x 1=\bmod \left(f i x\left(\operatorname{abs}(x 1-f i x(x 1)) \times 10^{14}\right), 4\right)+1$

Where, fix(.) is the rounding function, and $\operatorname{abs}($.$) is the$ absolute value function.

Step 2: The length of chains a(i) is determined with the following equation:

$$
\text { a (i) }=\left\{\begin{array}{l}
16, \text { if } x 1(i)=1 \\
8, \text { if } x 1(i)=2 \\
4, \text { if } x 1(i)=3 \\
2, \text { if } x 1(i)=4
\end{array}\right.
$$

Where, $\mathrm{i}=1,2,3 \ldots \mathrm{m}, \mathrm{m}$ is the size of the rows in the DNA sequence matrix.

Step 3: DNA dynamic chain matrices are obtained by decomposing each row of the DNA matrix sequences, according to different lengths, as shown in the following equation:

Row_chain $=$ DNA_decompose $\left(D N A \_m a t r i x(i,:), a(i)\right)$

Where DNA_decompose(.) is a generation function of the DNA dynamic chain, which means that the ith row in the DNA_matrix is decomposed into chains whose lengths are defined in a(i).

\section{Deletion Operation on the Dynamic DNA Chain}

Deletion operation on the DNA dynamic chains for each row or column is implemented using the chaotic sequence. The deletion operation function deletion $(\mathrm{A}, \mathrm{X})$ is defined as following:

In the function deletion(A, X), A is a chain set of a row, which can be represented as $A=\left\{a_{1}, a_{2}, a_{3} \ldots a_{n}\right\}$, where $\mathrm{n}$ is the number of chains. $\mathrm{a}_{\mathrm{i}}$ is the ith DNA chain. $\mathrm{X}$ is a chaotic sequence, which can be represented as $\mathrm{X}=\left\{\mathrm{x}_{1}, \mathrm{x}_{2}, \mathrm{x}_{3} \ldots \mathrm{x}_{\mathrm{n}}\right\} . \mathrm{x}_{\mathrm{i}}\left(\mathrm{x}_{\mathrm{i}} \in(0,1)\right)$ is the ith element in the chaotic sequence. Note that the length of the chaotic sequence and that of the DNA chain set are the same. Carry out deletion for $\mathrm{a}_{\mathrm{i}}$ when $\mathrm{x}_{\mathrm{i}}<0.5$, otherwise save the chain. Supposing that the $\mathrm{a}_{\mathrm{i}}$ chain has been deleted, the $\mathrm{a}_{\mathrm{i}}$ chain is moved to the end of the DNA chain. Other deleted chains can be processed in the same way. 


\section{Transposition Operation on the Dynamic DNA Chain}

Transposition operation on the DNA dynamic chains for each row or column is conducted using the chaotic sequence. The Transposition operation function Transposition(A, X) is defined as following:

In the function Transposition(A, X), the definitions of $\mathrm{A}$ and $\mathrm{X}$ are the same as those in section "Deletion Operation on the Dynamic DNA Chain." A new sequence $\mathrm{X}^{\prime}$ is obtained by transposing $X$, with $a_{i}$ and $a_{i^{\prime}}$ exchanged, where $i^{\prime}$ is the location of the ith element in $\mathrm{X}^{\prime}$.

\section{Insertion Operation on the Dynamic DNA Chain}

Insertion operation is used for the decryption process, the insertion operation function insertion $(\mathrm{A}, \mathrm{X})$ is defined as following:

In the function insertion $(A, X), A$ is a chain set of a row, which can be represented as $A=\left\{a_{1}, a_{2}, a_{3} \ldots a_{n}\right\}$ where $n$ is the number of chains. $a_{i}$ is the ith DNA chain. $X$ is a chaotic sequence, which can be represented as $\mathrm{X}=\left\{\mathrm{x}_{1}, \mathrm{x}_{2}, \mathrm{x}_{3} \ldots \mathrm{x}_{\mathrm{n}}\right\}$. $\mathrm{x}_{\mathrm{i}}\left(\mathrm{x}_{\mathrm{i}} \in(0,1)\right)$ is the ith element in the chaotic sequence. Note that the length of the chaotic sequence and that of the DNA chain set are the same. Set count $=0$, when $\mathrm{x}_{\mathrm{i}}<0.5$, carry out count $=$ count +1 , count is the number of deleted DNA chains in the encryption process. Carry out $e_{j}=a_{n-\text { count }+j}$, where $j=1,2, \ldots$ count, here $e_{j}$ is the $j$ th deleted DNA chain. Set $q=1, \mathrm{j}=1$, if $\mathrm{x}_{\mathrm{i}}<0.5, f_{i}=e_{j}, j++$; else $f_{i}=a_{q}, q++$. $\mathrm{A} 1=\left\{\mathrm{f}_{1}, \mathrm{f}_{2}, \mathrm{f}_{3} \ldots \mathrm{f}_{\mathrm{n}}\right\}$ is obtained after insertion operation.Other inserted chains can be processed in the same way.

\section{The Proposed Algorithm}

The detailed steps of the proposed algorithm are listed below.

Step 1: Input the initial values $\mathrm{x} 0, \mathrm{y} 0, \mathrm{z} 0$, w0 and an 8bit image $A(m, n)$, where $m$ and $n$ define the size of the image. A binary matrix $A^{\prime}(m, n \times 8)$ is obtained by transforming A $(\mathrm{m}, \mathrm{n})$.

Step 2: Use the SHA-256 function to generate the chaotic initial values x_s, y_s, z_s, w_s, as explained in section "Key Generation."

Step 3: Produce four chaotic sequences X, Y, Z, W using FOCHC with the initial values $x \_s, y \_s, z \_s, w \_s$, as detailed in section "Generation of FOCHC Sequences."

Step 4: Generate the matrix A_encode $(\mathrm{m}, \mathrm{n} \times 4)$ using the chaotic sequence $\mathrm{X} 1$ to encode $\mathrm{A}^{\prime}(\mathrm{m}, \mathrm{n} \times 8)$, as detailed in section "DNA Coding Rule." X1 is obtained using the following equation:

$$
X 1=\bmod \left(f i x\left(a b s(X-f i x(X)) \times 10^{14}\right), 8\right)+1
$$

Where fix(.) is the rounding function, and $\operatorname{abs}($.$) is the$ absolute value function.

Step 5: Scramble A_encode $(\mathrm{m}, \mathrm{n} \times 4)$ by using two chaotic sequences $\mathrm{Y}, \mathrm{Z}$, as explained in section "Scrambling of the DNA Sequence Matrix." This produces the matrix A_DNA_scrambling $(\mathrm{m}, \mathrm{n} \times 4)$.

Step 6: Calculate the hamming distance of A_DNA_scrambling to obtain the new initial values $\mathrm{x} \_\mathrm{h}, \mathrm{y} \_\mathrm{h}, \mathrm{z} \_\mathrm{h}, \quad \mathrm{w} \_\mathrm{h}$, as explained in section "Key
Generation by Hamming Distance," which are then used to generate four chaotic sequences $\mathrm{X}^{\prime}, \mathrm{Y}^{\prime}, \mathrm{Z}^{\prime}, \mathrm{W}^{\prime}$.

Step 7: Divide A_DNA_scrambling into different lengths of DNA dynamic chains row-by-row by using the chaotic sequence $\mathrm{X}^{\prime}$, as explained in section "Generation of the Dynamic DNA Chains." This produces the DNA chain matrix A_Row_Chain.

Step 8: Conduct the deletion and transposition operations on A_Row_Chain using the chaotic sequence $\mathrm{Y}^{\prime}$, as described in section "Deletion Operation on the Dynamic DNA Chain" and section "Transposition Operation on the Dynamic DNA Chain." After recombining the data, this produces the matrix A_Row_operation.

Step 9: Divide A_Row_operation into different lengths of DNA dynamic chains column-by-column using the chaotic sequence $Z^{\prime}$, as described in section "Generation of the Dynamic DNA Chains." This produces the DNA chain matrix A_Column_Chain.

Step 10: Conduct the deletion and transposition operations on A_Column_Chain using the chaotic sequence $\mathrm{W}^{\prime}$, as explained in section "Deletion Operation on the Dynamic DNA Chain" and section "Transposition Operation on the Dynamic DNA Chain.” After recombining the data, this produces the matrix A_Column_operation.

Step 11: Decode the matrix A_Column_Chain dynamically using the chaotic sequence W1, as explained in section "DNA Coding Rule." This produces the new matrix A_decode. W1 is calculated using the following equation:

$W 1=\bmod \left(f i x\left(\operatorname{abs}(W-f i x(W)) \times 10^{14}\right), 8\right)+1$

Step 12: Recombine A_decode to obtain the encrypted image $B$.

The decryption algorithm is the inverse of the encryption algorithm detailed above; also the delete operation needs to be replaced with the insert operation.

\section{SIMULATION RESULTS}

The proposed algorithm explained above is then tested on three kinds of medical images of MRI, CT, and X-ray. All of the experimental data are $512 \times 512$ images extracted from the database $^{1}$. Matlab 2019a is used to code the proposed algorithm, and the code is running in the 64-bit Window 7 environment with 8GB RAM and the i5-7200U CPU. The keys of the encryption algorithm presented in this article are composed of the hash value, the row and column hamming distance values, and two sets of chaotic initial values, as shown in Table 3. Table 4 lists the experimental results using the extracted images.

As illustrated in Table 4, no any useful information can be drawn from the encrypted images for all the three types of medical images tested, while the decrypted images show no difference when compared with the original images. From

${ }^{1}$ https://medpix.nlm.nih 
TABLE 3 | The key of the proposed algorithm.

\begin{tabular}{ll}
\hline $\begin{array}{l}\text { Composition of } \\
\text { the key }\end{array}$ & The key of encryption and decryption \\
\hline Hash value & $c 515 f 75 a 2 b 612 \mathrm{~d} 728 \mathrm{e} 3356 \mathrm{~b} 7 \mathrm{~b} 53925$ \\
& $32044172 \mathrm{~d} 647291 \mathrm{f} 10 \mathrm{f} 00075107 \mathrm{f} 161 \mathrm{bd} 9$ \\
Hamming distance & $\mathrm{R}_{\mathrm{h}}{ }^{\prime}=393425, \mathrm{C}_{\mathrm{h}}{ }^{\prime}=393058$ \\
Initial value of two & $\mathrm{x} 0=0.12, \mathrm{yO}=0.35, \mathrm{zO}=0.68, \mathrm{wO}=0.42$, \\
FOCHC system & $x 0^{\prime}=0.37, \mathrm{yO}^{\prime}=0.54, \mathrm{zO}=0.89, \mathrm{wO}^{\prime}=0.76$ \\
\end{tabular}

the point of view of visual inspection, the proposed algorithm worked satisfactorily.

\section{Security Analyses \\ Key Space Evaluation}

Section "Simulation Results" shows that the keys consist of three parts: the hash value, the hamming distances and the chaotic initial value. Therefore, in addition to the hash value and the hamming distances, there are another eight keys in the proposed algorithm. They are $\mathrm{x} 0, \mathrm{y} 0, \mathrm{z} 0, \mathrm{w} 0, \mathrm{x}^{\prime}, \mathrm{y}^{\prime}, \mathrm{z}^{\prime}$, and w0.' All of them have 14 bits precision, so the key space is $\left(10^{14}\right)^{7}=10^{112} \approx 2^{372}$. The SHA-256 value with the complexity of the finest attack is $2^{128}$. All of them are larger than $2^{100}$ (Wang X.Y. et al., 2020). Thus the key space is large enough to withstand BFA. Moreover, generation of the key depends on the original image, which forms a one-time pad shame, and makes it difficult for the attacker to predict the encryption key.

\section{Key Sensitivity Evaluation}

The keys are used in the encryption and decryption process, and the key sensitivity means that when the encryption keys each change slightly, the image generated will be completely different to the initial encrypted image. Similarly, when the decryption keys each change slightly, the correct decryption image cannot be obtained. This article tests the sensitivity of the keys from the aspects of encryption and decryption separately. Figure 6 and Table 5 show the results.

Figure 6B is the encrypted image of the "MRI-Knee-joint" with the keys listed in Table 3. Figure 6C is the same as Figure 6B beside $\mathrm{x} 0$ is changed to $\mathrm{x} 0+\mathrm{t}$, where $t=0.00000000000001$. Figure 6D shows that Figures 6B,C have much difference. To further observe the sensitivity of the keys, one key is changed slightly (add t) and the other keys kept unchanged, after that, these keys are used to encrypt the images "MRIknee," "CT-abdominal," and "X-ray-pelvic," respectively. At last, the difference rate of the encrypted images before and after the slight key changes is calculated. All the average difference rates in Table $\mathbf{5}$ are above $99.50 \%$, which is very close to $100 \%$. On the other hand, the original images are restored using three types of keys $\left(\mathrm{x} 0, \mathrm{R}_{\mathrm{h}}{ }^{\prime}\right.$ and the hash value) changed slightly. Figure $6 \mathrm{E}$ is the decrypted image with $\mathrm{x} 0=\mathrm{x} 0+\mathrm{t}$ and other keys unchanged. Figure $6 \mathrm{~F}$ is the decrypted image with $\mathrm{x} 0=\mathrm{R}_{\mathrm{h}}^{\prime}+1$ and other keys unchanged. Figure $6 \mathrm{G}$ is the decrypted image with the hash value + ' 1 ' and other keys unchanged. Figures 6 E-G show that the original image cannot be restored when the keys change slightly. The original image can only be recovered when the keys are correct, as shown in Figure $\mathbf{6 H}$. This test proved that the key sensitivity is very high, and the algorithm is robust against the exhaustive attacks.

\section{Statistical Analysis Evaluation \\ The Histogram Evaluation of the Decrypted Image}

The distribution of the histogram is evaluated by observing the histogram for the encrypted image and calculating the variance of the histogram. The more uniform the histogram distribution for the encrypted image, the stronger the ability of anti-statistical analysis. The variance of the histogram is defined as follow:

$$
\operatorname{Var}(M)=\frac{1}{n^{2}} \times \sum_{i=1}^{n} \sum_{j=1}^{n} \frac{1}{2}\left(m_{i}-m_{j}\right)^{2}
$$

Where $\mathrm{n}$ is the gray scale value, here it is set as $n=256 . \mathrm{m}_{\mathrm{i}}$ is the number of pixels whose gray values are equal to $i$, with $i$ being the value in the histogram value. $m_{i}$ is the same as $m_{j}$.

Figures 7A,C,E show the histogram of three kinds of original images, and Figures 7B,D,F show the histogram corresponding to the images after encrypting. Observation suggests that Figures 7B,D,F looks very uniform. In Table 6, the variance values for the encrypted images are significantly reduced. In addition, the average of the variance values is lower than other algorithm, as detail in the Table 7. In summary, it is difficult to extract original information through statistical analysis on the histogram.

\section{Correlation Coefficient Evaluation}

Usually, the recognizable images have high correlation, so correlation evaluation is one of the effective means to measure the encryption effect. The closer the correlation coefficient to 0 , the better the encryption result. The correlation coefficient is defined as follows:

$$
r_{x y}=\frac{\frac{1}{N} \times \sum_{i=1}^{N}\left(x_{i}-\frac{1}{N} \times \sum_{i=1}^{N} x_{i}\right)}{\left(y_{i}-\frac{1}{N} \times \sum_{i=1}^{N} y_{i}\right)}
$$

Where $x_{i}$ and $y_{i}$ are adjacent pixels selected randomly in three directions (horizontal, vertical, and diagonal). For evaluation 8,000 pairs of adjacent pixels are chosen for the test.

Figures $\mathbf{8 A - C}$ shows the correlation coefficients in the three directions, respectively. Obviously, the distribution of point sets is concentrated in the left subfigure of Figures $\mathbf{8 A - C}$. On the contrary, the distribution of point sets is discrete in the right subfigure of Figures 8A-C. The values of correlation coefficients are shown in Table 8. The correlation coefficients of the encrypted image are very close to 0 . From the comparison results, it is also better than other algorithms, which are shown in Table 9. This sufficiently demonstrates that it is difficult for attackers to obtain a cipher image by statistical pixel correlation. 
TABLE 4 | Results of the proposed algorithm.

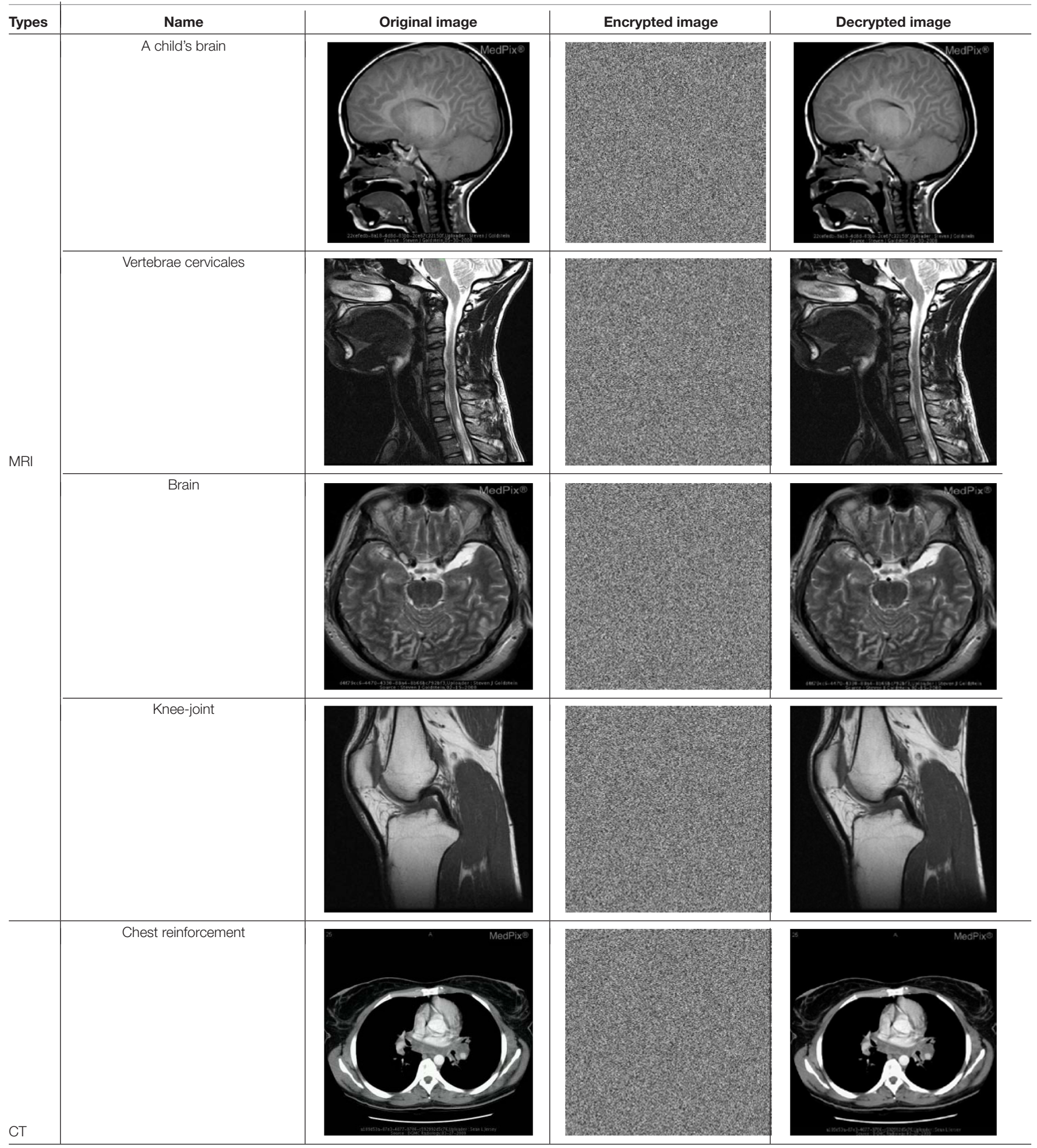


TABLE 4 | Continued

\begin{tabular}{|c|c|c|c|c|}
\hline Types & Name & Original image & Encrypted image & Decrypted image \\
\hline & Abdominal reinforcement & & & \\
\hline \multirow{2}{*}{ X-ray } & Chest & & & \\
\hline & Pelvic & & & \\
\hline
\end{tabular}
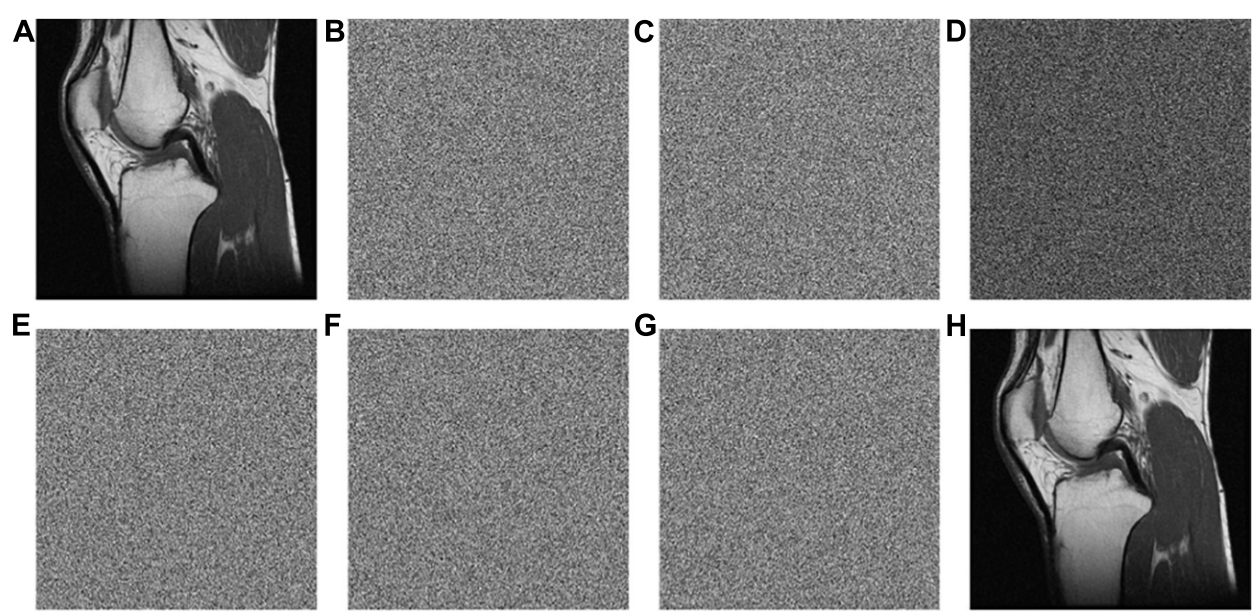

FIGURE 6 | Test of key sensitivity. (A) The original image of "MRI-knee joint." (B) The encrypted image with the initial key set. (C) The encrypted image with $x 0+t$. (D) The differential image between (B,C). (E) The decrypted image with xO+t. (F) The decrypted image with $R_{h}^{\prime}+1$. (G) The decrypted image with the hash value + "1." (H) The decrypted image with the correct key. 

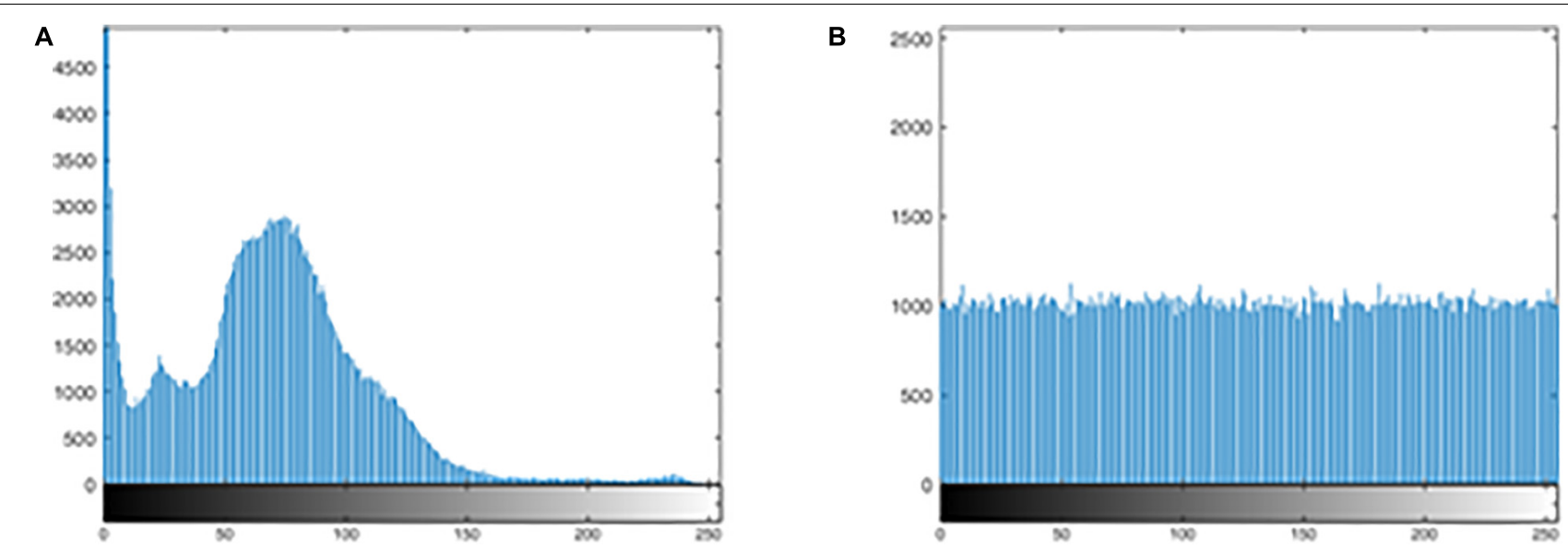

\section{C}

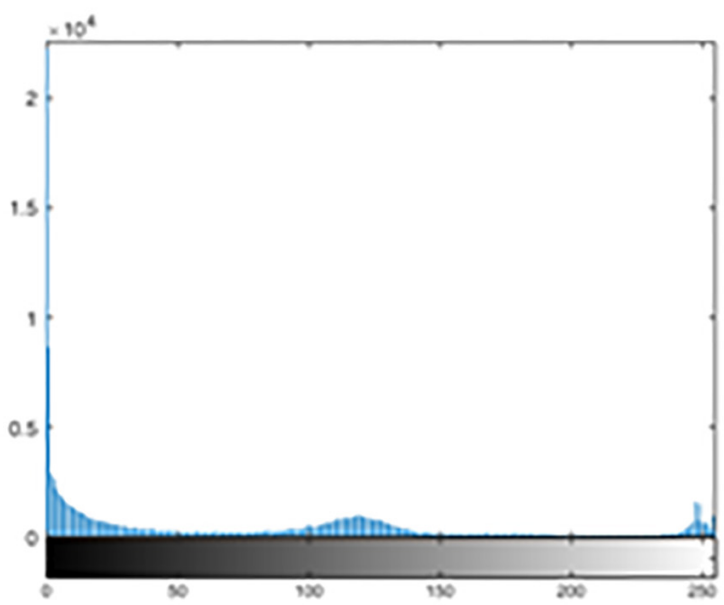

D

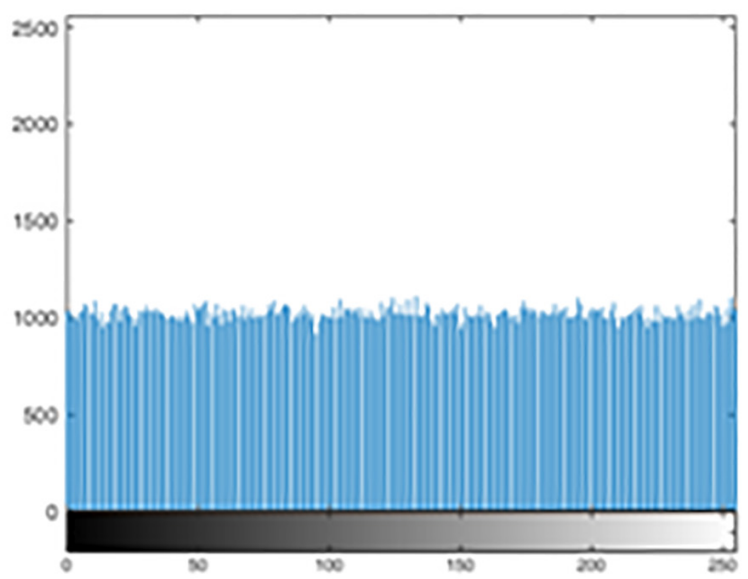

E

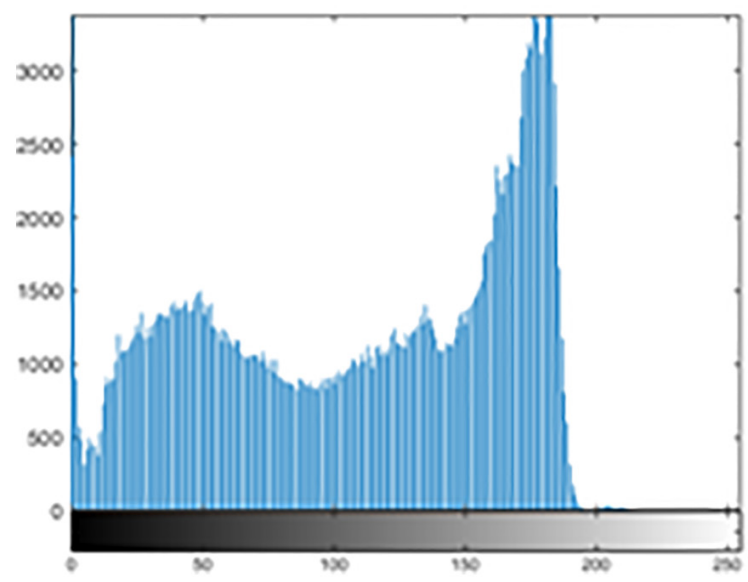

$\mathbf{F}$

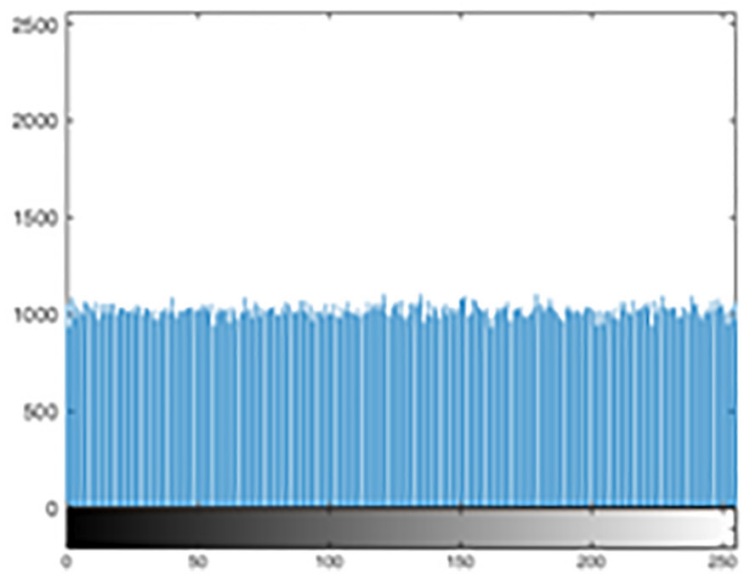

FIGURE 7 | The histogram of the original image and the encrypted image. (A) The histogram of the original image for "MRI-brain." (B) The histogram of the encrypted image for the "MRI-brain." (C) The histogram of the original image for the "CT-chest." (D) The histogram of the encrypted image for the "CT-chest." (E) The histogram of the original image for the "X-ray-chest." (F) The histogram of the encrypted image for the "X-ray-chest." 
TABLE 5 | Difference rate of two encrypted image obtained by slightly different keys.

\begin{tabular}{|c|c|c|c|c|c|c|c|c|c|}
\hline \multirow[t]{2}{*}{ Image } & \multicolumn{9}{|c|}{ Difference rate $(\%)$} \\
\hline & $x 0+t$ & $y 0+t$ & $\mathbf{z} 0+t$ & $w 0+t$ & $x 0^{\prime}+t$ & $y 0^{\prime}+t$ & $\mathrm{zO}^{\prime}+\mathrm{t}$ & $w 0^{\prime}+t$ & SHA-256+1 \\
\hline MRI-knee & 99.6101 & 99.6326 & 99.6025 & 99.6014 & 99.5232 & 99.5304 & 99.5224 & 99.5136 & 99.6132 \\
\hline CT-abdominal & 99.5964 & 99.6101 & 99.6307 & 99.6044 & 99.5209 & 99.4858 & 99.5049 & 99.5335 & 99.6120 \\
\hline X-ray-Pelvic & 99.6227 & 99.6174 & 99.6014 & 99.6106 & 99.5197 & 99.5182 & 99.5537 & 99.5308 & 99.6212 \\
\hline Average & 99.6097 & 99.6200 & 99.6115 & 99.6054 & 99.5220 & 99.5115 & 99.5270 & 99.5260 & 99.6155 \\
\hline
\end{tabular}

TABLE 6 | The variance of the histogram.

\begin{tabular}{lcc}
\hline Image & Original image & Encrypted image \\
\hline MRI-child's brain & $1.9859 \times 10^{7}$ & $1.0956 \times 10^{3}$ \\
MRI-cervical vertebra & $2.0992 \times 10^{6}$ & $1.0379 \times 10^{3}$ \\
MRI-brain & $2.8428 \times 10^{6}$ & $1.1222 \times 10^{3}$ \\
MRI-knee-joint & $2.6840 \times 10^{6}$ & 871.1016 \\
CT-chest & $8.0452 \times 10^{7}$ & $1.0736 \times 10^{3}$ \\
CT-abdominal & $4.4843 \times 10^{7}$ & 907.8516 \\
X-ray-chest & $7.8361 \times 10^{5}$ & $1.0098 \times 10^{3}$ \\
X-ray-pelvic & $2.0195 \times 10^{6}$ & 843.0469 \\
Average & $2.1938 \times 10^{7}$ & 995.1375 \\
\hline
\end{tabular}

TABLE 7 | The variance of the histogram comparison.

\begin{tabular}{lc}
\hline Algorithm & Variance \\
\hline Proposed & $\mathbf{9 9 5 . 1 3 7 5}$ \\
\hline Chai et al., 2019 & 1051 \\
Liu et al., 2019 & 1341 \\
\hline
\end{tabular}

\section{Global and Local Information Entropy Evaluation}

There is redundancy in any image, which is related to the probability or uncertainty of each pixel in the image. Usually, this uncertainty is measured with the global and the local information entropy (Zhang et al., 2012; Wu et al., 2013). The global information entropy is a measure of the distribution of all pixels in an image, while the local information entropy is a measure of the distribution of pixel values in an image block. Compared with the global information entropy, the local information entropy is more efficient, accurate and consistent in judging the pixel values distribution situation of the image.

It is known that the global information entropy of an ideal random image is 8. Also $\mathrm{Wu}$ et al., 2013 shows that the local entropy values for the ideal random image blocks of $16 \times 16$ and $32 \times 32$ are 7.1749 and 7.8087, respectively. Table 10 lists the global and local information entropy of all the encrypted images processed using the proposed algorithm. It is observed that the average global information entropy of all the encrypted image is 7.9993, and the average local entropy are 7.1715 for the $16 \times 16$ block and 7.8016 for the $32 \times 32$ block. All of them are close to the ideal values. Furthermore, comparison with other algorithms is shown in Table 11, which also includes the comparison of global information entropy corresponding to the image encryption algorithm. Apparently, the information entropy of the proposed algorithm in this study is superior to those for other algorithms.

\section{Plaintext Sensitivity (Differential Attack)}

Differential attack is one of the common attack methods used by cryptanalysts. Its main idea is to encrypt two original images with tiny change and no change, respectively, then compare the relationship between the encrypted image before and after change, and predict the encryption key, so as to decipher the encryption algorithm. NPCR and UACI in the Reference (Wang X.Y. et al., 2020) are used here to test the ability of the algorithms to resist differential attack.

The NPCR and UACI values of the encrypted images obtained from the two slightly changed images are shown in Table 12. The average values obtained are $99.6191 \%$ and $33.4815 \%$,
A

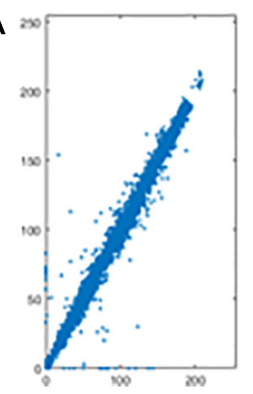

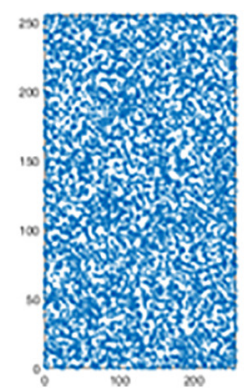

B

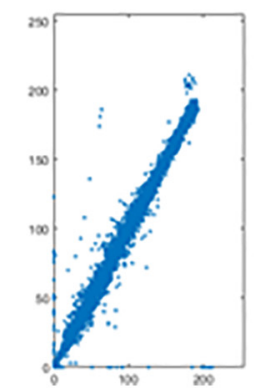

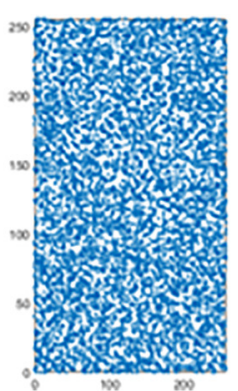

C

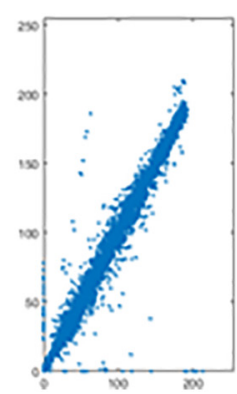

FIGURE 8 | Correlation of adjacent pixels of the original image and the encrypted image for "CT-chest." (A) Horizontal direction. (B) Vertical direction. (C) Diagonal direction. 
TABLE 8 | Correlation coefficients of two adjacent pixels of the original and the encrypted images.

\begin{tabular}{|c|c|c|c|c|c|c|}
\hline \multirow[t]{2}{*}{ Image } & \multicolumn{3}{|c|}{ Original image } & \multicolumn{3}{|c|}{ Encrypted image } \\
\hline & $\mathbf{H}$ & $\mathbf{v}$ & D & $\mathbf{H}$ & $\mathbf{v}$ & D \\
\hline MRI-child's brain & 0.9757 & 0.9806 & 0.9563 & 0.0013 & $6.5787 \times 10^{-4}$ & -0.0049 \\
\hline MRI-cervical vertebra & 0.9714 & 0.9738 & 0.9495 & $-1.6397 \times 10^{-4}$ & $9.0951 \times 10^{-4}$ & -0.0015 \\
\hline MRI-brain & 0.9812 & 0.9809 & 0.9607 & 0.0036 & -0.0075 & 0.0021 \\
\hline MRI-knee-joint & 0.9928 & 0.9970 & 0.9912 & $-9.6693 \times 10^{-4}$ & $-6.6741 \times 10^{-4}$ & $4.7456 \times 10^{-4}$ \\
\hline CT-chest & 0.9746 & 0.9603 & 0.9455 & 0.0012 & $-2.7242 \times 10^{-4}$ & 0.0011 \\
\hline CT-abdominal & 0.9788 & 0.9827 & 0.9656 & $-9.1875 \times 10^{-4}$ & $3.8182 \times 10^{-4}$ & -0.0018 \\
\hline X-ray-chest & 0.9946 & 0.9779 & 0.9774 & 0.0011 & $6.4868 \times 10^{-4}$ & 0.0049 \\
\hline X-ray-pelvic & 0.9340 & 0.9529 & 0.9114 & 0.0012 & $-2.0257 \times 10^{-4}$ & $-4.7550 \times 10^{-4}$ \\
\hline
\end{tabular}

TABLE 9 | Correlation coefficients comparison.

\begin{tabular}{lccc}
\hline Algorithm & H & V & D \\
\hline Proposed (CT-chest) & $\mathbf{0 . 0 0 1 2}$ & $-\mathbf{0 . 0 0 0 3}$ & $\mathbf{0 . 0 0 1 1}$ \\
\hline Hossein et al., 2018 (medical image) & 0.0031 & 0.0029 & 0.0013 \\
Belazi et al., 2019 (medical image) & 0.0013 & -0.0049 & 0.0057 \\
Dagadu et al., 2019a (medical image) & -0.0016 & 0.0043 & -0.0061 \\
Wang X.Y. et al., 2020 & -0.0021 & 0.0009 & 0.0003 \\
Hossein et al., 2020 & 0.0059 & 0.0029 & 0.0018 \\
Wu et al., 2019 & 0.0158 & 0.0023 & -0.0336 \\
Zhang X.C. et al., 2017 & 0.0082 & 0.0032 & 0.0150
\end{tabular}

TABLE 10 | The entropy of eight medical encrypted images.

\begin{tabular}{lccc}
\hline Image & Entropy & $\begin{array}{c}\text { Local entropy } \\
\mathbf{( 1 6} \times \mathbf{1 6})\end{array}$ & $\begin{array}{c}\text { Local entropy } \\
\mathbf{( 3 2} \times \mathbf{3 2})\end{array}$ \\
\hline MRI-child's brain & 7.9992 & 7.1530 & 7.7962 \\
MRI-Vertebrae cervicales & 7.9993 & 7.1705 & 7.7995 \\
MRI-Brain & 7.9992 & 7.1800 & 7.8037 \\
MRI-Kneejoint & 7.9994 & 7.1935 & 7.8010 \\
CT-Chest & 7.9993 & 7.1688 & 7.8034 \\
CT-Abdominal & 7.9994 & 7.1567 & 7.8007 \\
X-ray-Chest & 7.9993 & 7.1615 & 7.8037 \\
X-ray-Pelvic & 7.9994 & 7.1883 & 7.8046 \\
Average & 7.9993 & 7.1715 & 7.8016 \\
\hline
\end{tabular}

which are higher than the values for other algorithms, as detailed in Table 13.

\section{Noise Attack}

Images can be contaminated by noise during transmission. To analyze the anti-noise capability, the same encrypted image is attacked by the salt and the pepper noise with the density of 0.002 , $0.005,0.05,0.1,0.25$, and 0.5, respectively, Figures 9A-F lists the decrypted images after being attacked with the salt and the pepper noise. All of them can clearly show the outline and texture of the original image. Further, equation (17) is used to calculate the PSNR between the original image and Figures 9A-F. The PSNR of the proposed algorithm is then compared with that of other algorithms. Results in Figure $\mathbf{9}$ and Table $\mathbf{1 4}$ show that the
TABLE 11 | Global information entropy comparison.

\begin{tabular}{lc}
\hline Algorithm & Entropy \\
\hline Proposed & $\mathbf{7 . 9 9 9 3}$ \\
\hline Hossein et al., 2018 (medical image) & 7.9990 \\
Belazi et al., 2019 (medical image) & 7.9974 \\
Dagadu et al., 2019a (medical image) & 7.9993 \\
Hua et al., 2018 (medical image) & 7.9981 \\
Wang X.Y. et al., 2020 & 7.9971 \\
Azimi and Ahadpour, 2020 & 7.9988 \\
Hossein et al., 2020 & 7.9989 \\
Wu et al., 2019 & 7.99895 \\
Yang et al., 2019 & 7.9964 \\
\hline
\end{tabular}

TABLE 12 | The result of differential attack (NPCR, UACI).

\begin{tabular}{lcc}
\hline Image & NPCR (\%) & UACI (\%) \\
\hline MRI-child's brain & 99.6124 & 33.4986 \\
MRI-Vertebrae cervicales & 99.6162 & 33.4415 \\
MRI-Brain & 99.6185 & 33.4285 \\
MRI-Knee joint & 99.6220 & 33.4547 \\
CT-Chest & 99.6254 & 33.4474 \\
CT-Abdominal & 99.6334 & 33.5747 \\
X-ray-Chest & 99.6059 & 33.4359 \\
X-ray-Pelvic & 99.6193 & 33.5704 \\
Average & 99.6191 & 33.4815 \\
\hline
\end{tabular}

proposed algorithm is immune to the salt and the pepper noise.

$$
P S N R=10 \lg \frac{255 \times 255 \mathrm{MN}}{\sum_{i=1}^{M} \sum_{j=1}^{N}\left|x^{\prime}(i, j)-x(i, j)\right|^{2}}
$$

\section{Occlusion Attack}

To analyze the anti-occlusion capability of the proposed algorithm, the same cipher image is occluded with $1 / 16,1 / 8,1 / 4$, and $1 / 2$, respectively. Then, the blocked images are decrypted with the proposed algorithm. Take the image "CT-chest" as the example, which are shown in Figures 10A-H. As shown, all the encrypted images which are occluded with different area are recovered successfully. In all of them the information of the 
TABLE 13 | Comparison of the average differential attack (NPCR,UACI) by different encryption algorithms.

\begin{tabular}{lcc}
\hline Algorithm & NPCR (\%) & UACI (\%) \\
\hline Proposed & $\mathbf{9 9 . 6 1 9 1}$ & $\mathbf{3 3 . 4 8 1 5}$ \\
\hline Hossein et al., 2018 (medical image) & 99.1349 & 33.1633 \\
Belazi et al., 2019 (medical image) & 99.6536 & 33.4121 \\
Dagadu et al., 2019a (medical image) & 99.6100 & 33.5075 \\
Wang X.Y. et al., 2020 & 99.5956 & 33.4588 \\
Wang et al., 2018 & 99.5700 & 32.3800 \\
Hossein et al., 2020 & 99.5438 & 33.4742 \\
Wu et al., 2019 & 99.5666 & 33.3966 \\
Yang et al., 2019 & 99.6105 & 33.4694
\end{tabular}

original image can be identified. Additionally, comparison of the ability to resist occlusion attack for the proposed and other algorithm by PSNR is shown in Table 15. Obviously the proposed algorithm is superior to others.

\section{Known-Plaintext and Chosen-Plaintext Attacks}

Kerckhoffs' principles in cryptography state that encryption and decryption algorithms are known or transparent in a cryptosystem. Therefore, the security of the cryptosystem
TABLE 14 | PSNR (db) between the original and the decrypted images under noise.

\begin{tabular}{lcccccc}
\hline \multirow{2}{*}{ Algorithm } & \multicolumn{5}{c}{ Density of salt \& pepper noise } \\
\cline { 2 - 7 } & $\mathbf{0 . 0 0 2}$ & $\mathbf{0 . 0 0 5}$ & $\mathbf{0 . 0 5}$ & $\mathbf{0 . 1}$ & $\mathbf{0 . 2 5}$ & $\mathbf{0 . 5}$ \\
\hline Proposed & $\mathbf{3 3 . 8 8 7 0}$ & $\mathbf{2 9 . 6 6 8 1}$ & $\mathbf{1 9 . 7 3 5 3}$ & $\mathbf{1 6 . 6 3 5 3}$ & $\mathbf{1 2 . 4 5 5 2}$ & $\mathbf{9 . 1 9 5 1}$ \\
\hline $\begin{array}{l}\text { Belazi et al., } \\
\text { 2019 }\end{array}$ & 32.8396 & 28.7068 & 18.8395 & 15.8599 & 12.2262 & 9.8903 \\
$\begin{array}{l}\text { Zhou et al., } \\
\text { 2015 }\end{array}$ & 26.1682 & 21.9976 & 12.8812 & 10.6900 & 8.8973 & 8.5504 \\
Hua and Zhou, \\
2017 & 8.5900 & 8.5625 & 8.5514 & 8.5476 & 8.5454 & 8.5428 \\
Hua et al., 2018 & 29.8380 & 25.6571 & 15.8923 & 13.1335 & 10.2166 & 8.8271 \\
Liu et al., 2016 & $/$ & 19.1553 & 19.5829 & 11.9524 & $/$ & $/$ \\
\hline
\end{tabular}

depends on the key rather than the encryption algorithm itself. By exploring the relationship between the key and the ciphertext or the plaintext and ciphertext, the attacker obtains the valid equivalent key, and then decrypts the original image. The main methods include the ciphertext-only attack, KPA, CPA, and chosen ciphertext attack (CCA). Among these attack methods, $\mathrm{CPA}$ is recognized as the strongest attack method, so the ability of the current algorithm to resist CPA is analyzed here.

As for the key generation, the encryption keys of this algorithm are generated by the SHA-256 function and the
A

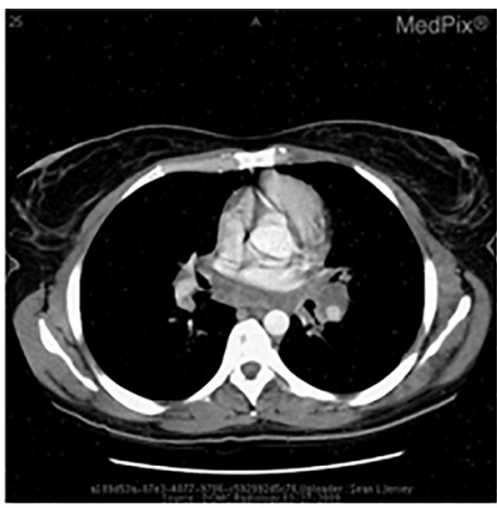

B

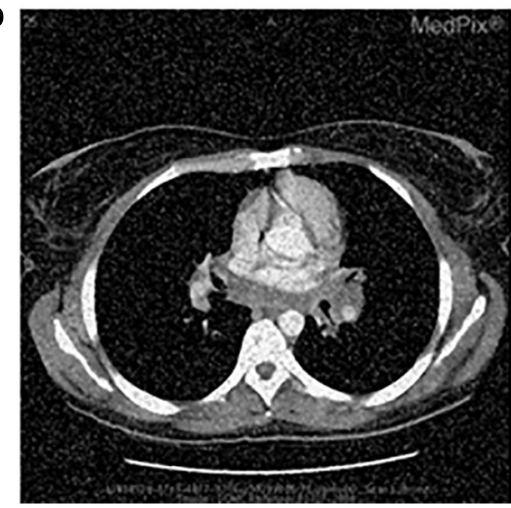

E

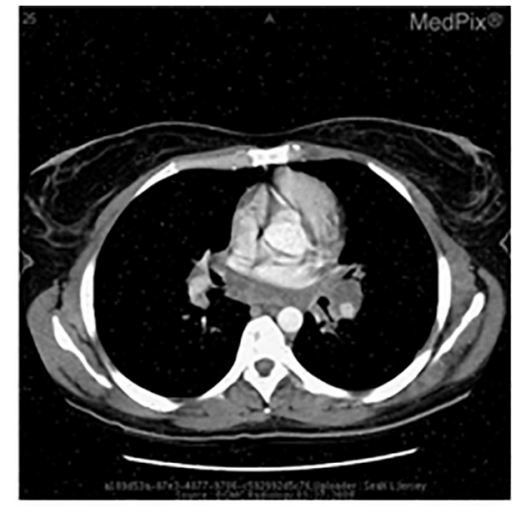

C
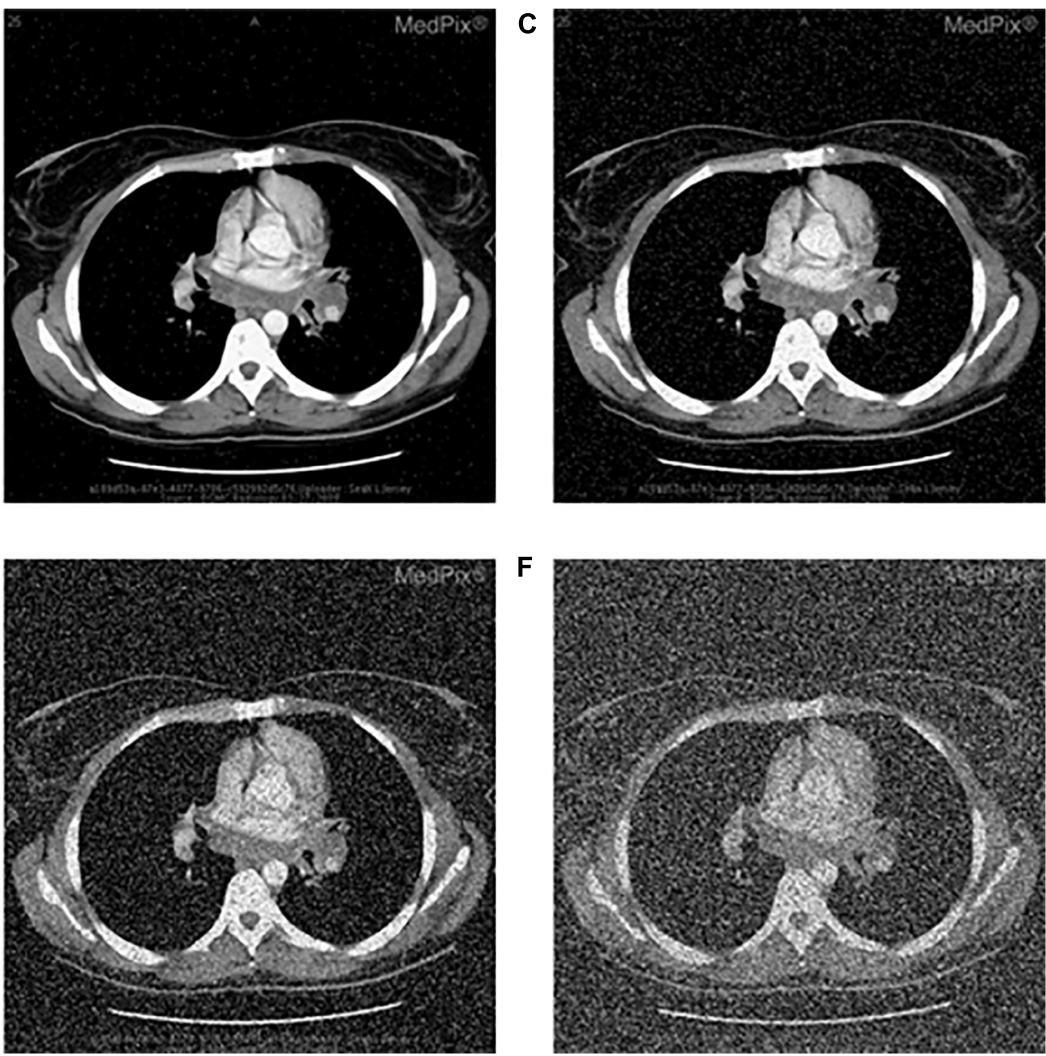

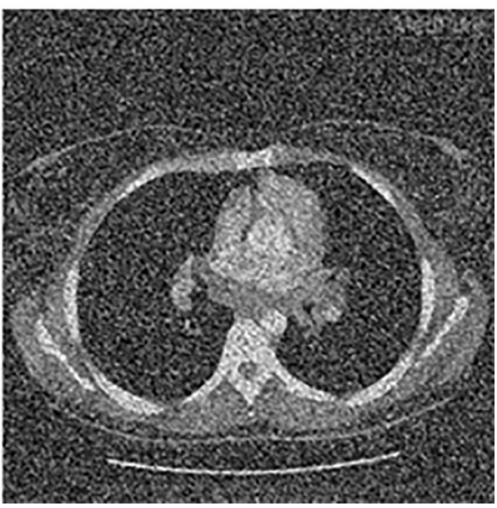

FIGURE 9 | Salt-and-pepper noise attack of encrypted CT-chest, decrypted image with density value (A) 0.002, (B) 0.005, (C) 0.05, (D) 0.1, (E) 0.25, and (F) 0.5. 

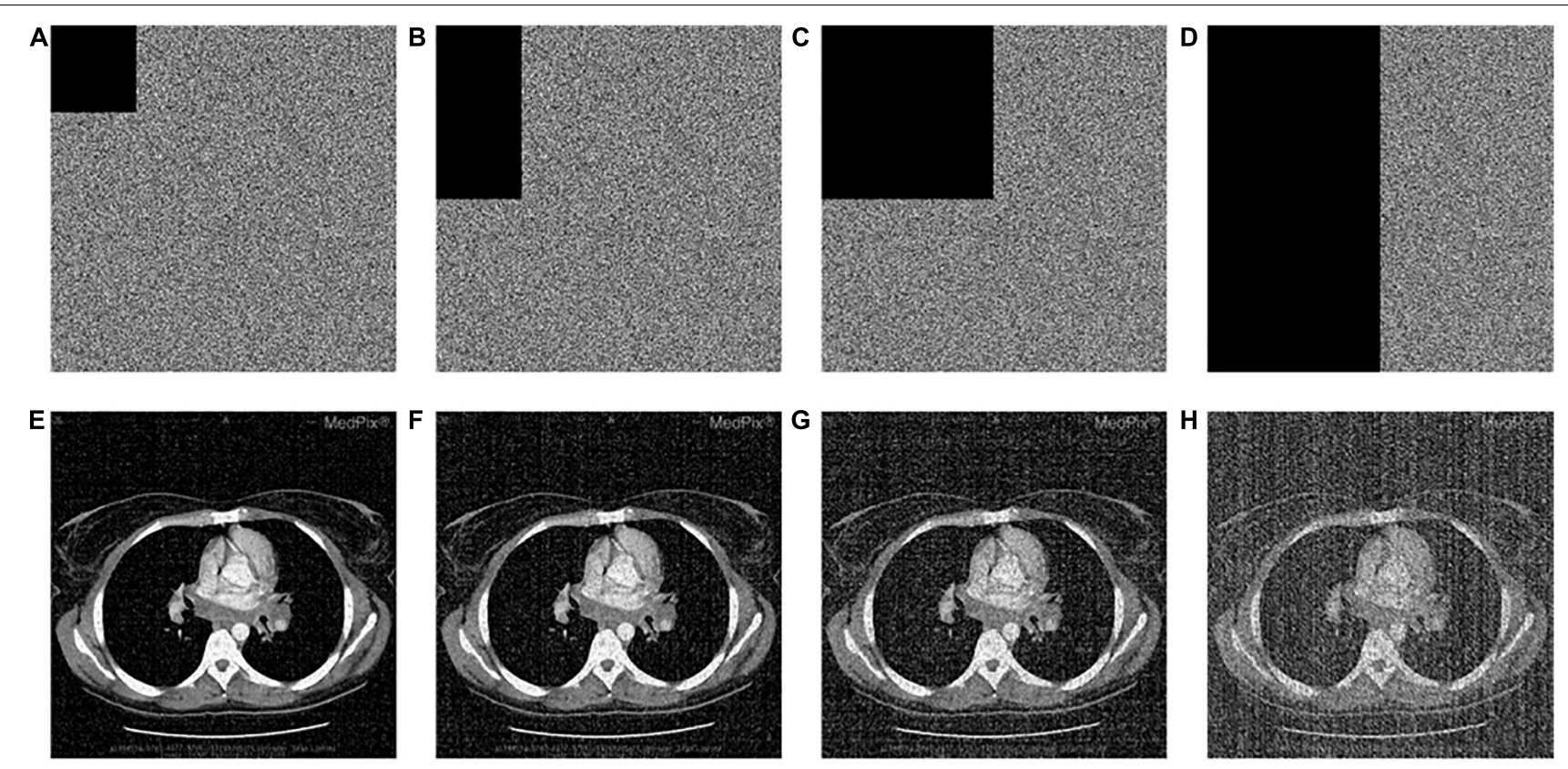

FIGURE 10 | Occlusion attack: the data loss of encrypted "CT-chest" with (A) 1/16, (B) 1/8, (C) 1/4, and (D) 1/2; corresponding decrypted image (E-H) in accordance with (A-D)

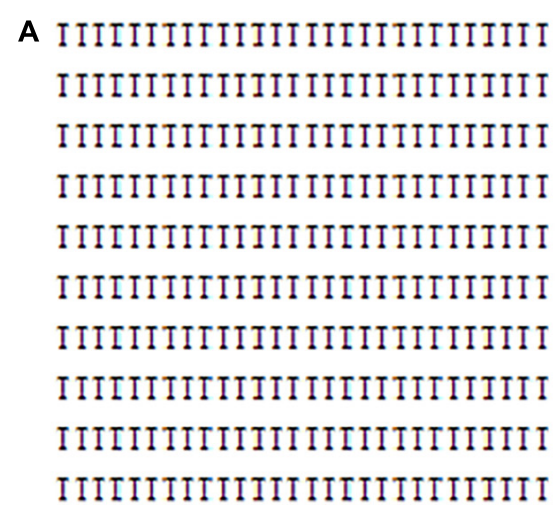

B CAAICGIGICGAIIIGGCAGCGGCAAGAG AAAGGGGCICAAGCCGIAAIGCGCGAGAI AIACAICIIGICAIG ICCIAIAGGAIGIG ACAGCCAGIIAAG IAGACAIACCIGIICG AG IGCIGAACG ICCCCG IACGCCGG IAAG II IAG ICI II IG I IAICGGGGAACGATAI AII ICAG ICCACIACIICGAAAGGGGAGA IGACACG ICCACAIGCIGAGCACG ITGCI IGG IGCAICCCACGGCGGIGGGAAAIGG IAAGCI IAICIGGCAAG IGGAGAACIAAC

FIGURE 11 | The part of DNA encoding matrix of "pure white" image: (A) DNA fixed encoding (B) DNA dynamic encoding of the proposed algorithm.
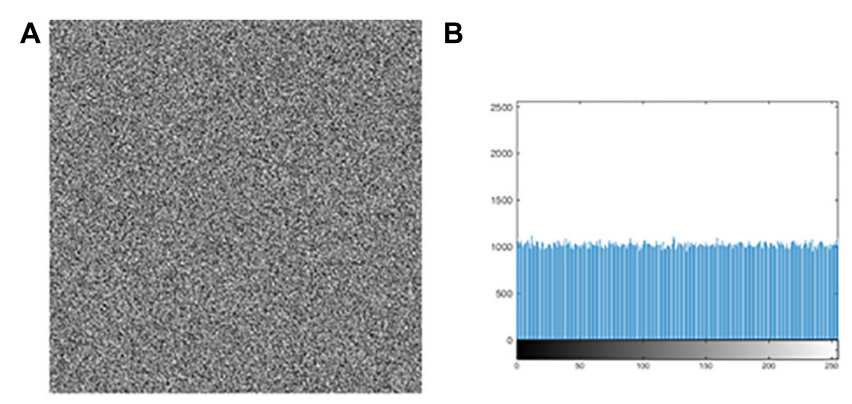

C

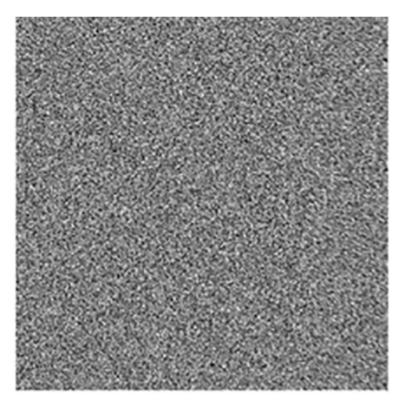

D

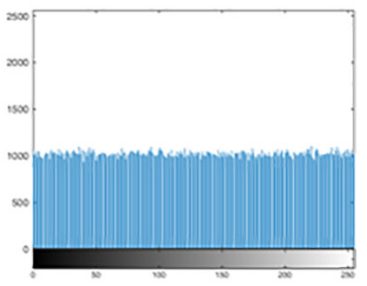

FIGURE 12 | The encrypted image and histogram of "pure white" image and "pure black" image: (A) The encrypted image of "pure white." (B) The histogram of (A). (C) The encrypted image of "pure black." (D) The histogram of (C). 
TABLE 15 | PSNR (db) between the original and decrypted images under occlusion.

\begin{tabular}{lcccc}
\hline Algorithm & \multicolumn{4}{c}{ Occlusion } \\
\cline { 2 - 5 } & $\mathbf{1 / 1 6}$ & $\mathbf{1 / 8}$ & $\mathbf{1 / 4}$ & $\mathbf{1 / 2}$ \\
\hline proposed & $\mathbf{1 8 . 7 2 7 5}$ & $\mathbf{1 5 . 6 4 4 5}$ & $\mathbf{1 2 . 3 9 4 7}$ & $\mathbf{9 . 1 3 1 7}$ \\
Belazi et al., 2019 & 26.6301 & 17.6447 & 14.6193 & 11.6147 \\
Zhou et al., 2015 & 12.0881 & 10.0969 & 8.8968 & 8.5539 \\
Hua and Zhou, 2017 & 8.5675 & 8.5540 & 8.5502 & 8.5480 \\
Hua et al., 2018 & 17.7218 & 14.8610 & 12.1275 & 9.7698 \\
\hline
\end{tabular}

hamming distances. Because the calculation of the SHA-256 function and the hamming distances are closely related to the plaintext, the key is very sensitive to the plaintext. In other words, a small change in the plaintext image produces a completely different key, as detailed in section "Key Sensitivity Evaluation." In the DNA encoding, this article uses the DNA dynamic encoding by binary bit, compared with the traditional fixed DNA coding and other existing DNA dynamic encoding methods, the base distribution is more uniform, which can be found in Table 2 . Additionally, for pure white or pure black images, encoding with DNA fixed can cause multiple base repeats, as shown in Figure 11. Clearly, Figure 11 A gives an attacker an opportunity, but the DNA bases in Figure 11B are irregular. From this whole encryption system, both "pure white" and "pure black" images of the encrypted images and the corresponding histograms are derived, which are shown in Figure 12. Moreover, Figures 12A,C are evaluated in Table 16. The histogram in Figure 12 is evenly distributed. The information entropy in Table 16 is 7.9994. Their NPCR and UACI are both higher than $99.6 \%$ and $33.4 \%$. The correlation coefficients are close to 0 . It can be shown that it is difficult for an attacker to analyze the equivalent key by choosing pure white or black images. To sum up, the proposed algorithm is robust in defending against the chosen plaintext attack.

\section{Randomness Detection}

Randomness detection examines whether the detected sequence demonstrates the characteristics of the random sequence, using the techniques of probability statistics. The most authoritative package for the randomness test is the Special Publication 800-22, provided by the National Institute of Standards and Technology (NIST) of the United States (Khawaja and Khan, 2019). This test package uses the P-value returned for different aspects of the evaluation process for making the judgment. Only when each P-value is greater than 0.01 , the test sequence is recognized as a random sequence. In this study, the randomness of the encrypted image of "MRI-Brain" is examined here as an example, and the results are shown in Table 17. The results of all the test items in Table $\mathbf{1 7}$ are "success," which proves that the
TABLE 17 | NIST randomness test of encrypted images.

\begin{tabular}{lcc}
\hline Test & $\boldsymbol{P}$-values & Results \\
\hline Frequency & 0.139830 & Success \\
Block frequency & 0.747300 & Success \\
Rank & 0.944274 & Success \\
Run $(M=10,000)$ & 0.240022 & Success \\
long runs of ones & 0.937168 & Success \\
Linear complexity & 0.618749 & Success \\
Overlapping templates & 0.446549 & Success \\
Non-overlapping templates & all $P$-value $>0.01$ & Success \\
FFT & 0.967619 & Success \\
Approximate entropy & 0.801709 & Success \\
Universal & 0.507906 & Success \\
Serial P values 1 & 0.275633 & Success \\
Serial P values 2 & 0.295743 & Success \\
Cumulative sums forward & 0.168961 & Success \\
Cumulative sums reverse & 0.075333 & Success \\
Random excursions & all $P$-value $>0.01$ & Success \\
Random excursions variant & all $P$-value $>0.01$ & Success \\
\hline
\end{tabular}

TABLE 18 | Comparison of efficiency.

\begin{tabular}{lc}
\hline Algorithm & Complexity \\
\hline Proposed & $\mathrm{O}(41 \mathrm{MN}+5 \mathrm{M}+2 \mathrm{NN})$ \\
Hua and Zhou, 2017 & $\mathrm{O}(108 \mathrm{MN}+72 \mathrm{~L} 4)$ \\
Sun, 2018 & $\mathrm{O}(579 \mathrm{MN})$ \\
Belazi et al., 2019 & $\mathrm{O}(124 \mathrm{MN})$ \\
\hline
\end{tabular}

encrypted image obtained by using the proposed algorithm has good randomness.

\section{Efficiency of the Proposed Algorithm}

The efficiency of the algorithm is determined by the time expense of the algorithm. The time cost of the proposed algorithm is $\mathrm{O}(41 \mathrm{MN}+5 \mathrm{M}+20 \mathrm{~N})$. Table $\mathbf{1 8}$ lists the results comparison with other algorithms. From Table 18, it is concluded that the encryption efficiency of the proposed algorithm is higher than other ones in the literature.

\section{CONCLUSION}

For medical images with large storage space and high pixel redundancy, the encryption effect, security and efficiency of encryption algorithm should have higher standards. The proposed algorithm combines the SHA-256 and the hamming distances to obtain the keys, uses the excellent FHCOC system to realize the best DNA dynamic coding, to generate the DNA dynamic chains of different lengths, to carry out dynamic deletion

TABLE 16 | The performance of the encrypted "pure white" image and the encrypted "pure black" image.

\begin{tabular}{|c|c|c|c|c|c|c|c|}
\hline Encrypted image & Entropy & variance & Horizontal & Vertical & Diagonal & NPCR & UACI \\
\hline White & 7.9994 & 816.5000 & 0.0018 & -0.0014 & 0.0016 & 99.6078 & 33.4375 \\
\hline Black & 7.9994 & 880.3828 & 0.0029 & -0.0013 & -0.0037 & 99.6136 & 33.5343 \\
\hline
\end{tabular}


operation and dynamic transposition operation of DNA chains. Test results show that the full diffusion of bases causes the pixels of medical images to be completely disorganized; the efficiency is higher and can resist all common attacks. Of course, the proposed algorithm is not only suitable for medical image encryption, but also suitable for other image encryption scenarios. For future research, the proposed algorithm can be applied to large storage space and parallelism of DNA computing for the protection of medical images.

\section{DATA AVAILABILITY STATEMENT}

The original contributions presented in the study are included in the article/supplementary material, further inquiries can be directed to the corresponding author.

\section{REFERENCES}

Adleman, L. M. (1994). Molecular computation of solutions to combinatorial problems. Science 266, 1021-1024. doi: 10.1126/science.7973651

Akhavan, A., Samsudin, A., and Akhshani, A. (2017). Cryptanalysis of an image encryption algorithm based on DNA encoding. Optics Laser Technol. 95, 94-99. doi: 10.1016/j.optlastec.2017.04.022

Azimi, Z., and Ahadpour, S. (2020). Color image encryption based on DNA encoding, and pair coupled chaotic maps. Multimedia Tools Appl. 79, 17271744. doi: 10.1007/s11042-019-08375-6

Belazi, A., Hermassi, H., Rhouma, R., and Belghith, S. (2014). Algebraic analysis of a RGB image encryption algorithm based on DNA encoding, and chaotic map. Nonlin. Dyn. 76, 1989-2004. doi: 10.1007/s11071-014-1263-y

Belazi, A., Talha, M., Kharbech, S., and Xiang, W. (2019). Novel medical image encryption scheme based on chaos, and DNA encoding. IEEE Access. 7, $36667-$ 36681. doi: 10.1109/ACCESS.2019.2906292

Chai, X. L., Gan, Z. H., Yang, K., Chen, Y. R., and Liu, X. X. (2017). An image encryption algorithm based on the memristive hyperchaotic system, cellular automata, and DNA sequence operation. Signal Process. 52, 6-19. doi: 10.1016/ j.image.2016.12.007

Chai, X. L., Gan, Z. H., Yuan, K., Chen, Y. R., and Liu, X. X. (2019). A novel image encryption scheme based on DNA sequence operations, and chaotic systems. Neural Comput. Appl. 31, 219-237. doi: 10.1007/s00521-017-2993-9

Dagadu, J. C., Li, J. P., and Aboagye, E. O. (2019a). Medical image encryption based on hybrid chaotic DNA diffusion. Wireless Pers. Commun. 108, 591-612. doi: 10.1007/s11277-019-06420-z

Dagadu, J. C., Li, J. P., Aboagye, E. O., and Deynu, F. K. (2019b). Medical image encryption scheme based on multiple chaos, and DNA coding. Int. J. Netw. Secur. 21, 83-90.

Donato, C., and Giuseppe, G. (2008). Bifurcation, and chaos in the fractional-order Chen system via a time-domain approach. Int. J. Bifurc. Chaos 18, 1845-1863. doi: 10.1142/S0218127408021415

Dou, Y. Q., Liu, X. M., Fan, H. J., and Li, M. (2017). Cryptanalysis of a DNA, and chaos based image encryption algorithm. Optik 145, 456-464. doi: 10.1016/j. ijleo.2017.08.050

Hermassi, H., Belazi, A., and Rhouma, R. (2014). Security analysis of an image encryption algorithm based on a DNA addition combining with chaotic maps. Multimedia Tools Appl. 72, 2211-2224. doi: 10.1007/s11042-013-1533-6

Hossein, N., Rasul, E., Homayun, M., Frederico, G. G., and Vitor, N. C. (2018). Medical image encryption using a hybrid model of modified genetic algorithm, and coupled map lattices. Optics Lasers Eng. 110, 24-32. doi: 10.1016/j. optlaseng.2018.05.009

Hossein, N., Rasul, E., Mehdi, Y., Malrey, L., and Gisung, J. (2020). Binary search tree image encryption with DNA. Optik 202:163505. doi: 10.1016/j.ijleo.2019. 163505

\section{AUTHOR CONTRIBUTIONS}

XLX and CJZ: conceptualization. XLX: methodology. HYJ and DSZ: formal analysis. XLX: investigation and writing - original draft preparation. HYJ and CJZ: writing - review and editing. DSZ and CJZ: funding acquisition. All authors have read and agreed to the published version of the manuscript.

\section{FUNDING}

This work was supported in part by the National Natural Science Foundation of China under the grant number 61672121, in part by the program for the Liaoning Distinguished Professor, the Science and Technology Innovation Fund of Dalian (No. 2018J12GX036).

Hua, Z., Yi, S., and Zhou, Y. (2018). Medical image encryption using high-speed scrambling, and pixel adaptive diffusion. Signal. Process. 144, 134-144. doi: 10.1016/j.sigpro.2017.10.004

Hua, Z., and Zhou, Y. (2017). Design of image cipher using block-based scrambling, and image filtering. Inf. Sci. 396, 97-113. doi: 10.1016/j.ins.2017. 02.036

Kalpana, J., and Murali, P. (2015). An improved color image encryption based on multiple DNA sequence operations with DNA synthetic image, and chaos. Optik 126, 5703-5709. doi: 10.1016/j.ijleo.2015.09.091

Khawaja, M. A., and Khan, M. (2019). Application based construction, and optimization of substitution boxes over 2D mixed chaotic maps. Int. J. Theor. Phys. 58, 3091-3117. doi: 10.1007/s10773-019-04188-3

Kumar, M., Iqbal, A., and Kumar, P. (2016). A new RGB image encryption algorithm based on DNA encoding, and elliptic curve Diffie-Hellman cryptography. Signal Process. 125, 187-202. doi: 10.1016/j.sigpro.2016.01.017

Li, T. Y., Yang, M. G., Wu, J., Jing, X., and Elsaid, A. (2017). A novel image encryption algorithm based on a fractional-order hyperchaotic system, and DNA computing. Complexity 2017, 1-13. doi: 10.1155/2017/9010251

Li, X., Wang, B., Lv, H., Yin, Q., Zhang, Q., and Wei, X. P. (2020). Constraining DNA sequences with a triplet-bases unpaired. IEEE Trans. Nanobiosci. 19, 299-307. doi: 10.1109/TNB.2020.2971644

Liu, C. J., Liu, Y., Zhu, E. Q., and Zhang, Q. (2020). Cross-inhibitor: a time-sensitive molecular circuit based on DNA strand displacement. Nucleic Acids Res. 48, 10691-10701. doi: 10.1093/nar/gkaa835

Liu, H., Zhao, B., and Huang, L. Q. (2019). A remote-sensing image encryption scheme using DNA bases probability, and two-dimensional logistic map. IEEE Access. 7, 65450-65459. doi: 10.1109/ACCESS.2019.2917498

Liu, L. L., Zhang, Q., and Wei, X. P. (2012). A RGB image encryption algorithm based on DNA encoding, and chaos map. Comput. Electr. Eng. 38, 1240-1248. doi: 10.1016/j.compeleceng.2012.02.007

Liu, Y., Wang, J., Fan, J. H., and Gong, L. H. (2016). Image encryption algorithm based on chaotic system, and dynamic S-boxes composed of DNA sequences. Multimedia Tools Appl. 75, 4363-4382. doi: 10.1007/s11042-015-2479-7

Liu, Y. S., Tang, J., and Xie, T. (2014). Cryptanalyzing a RGB image encryption algorithm based on DNA encoding, and chaos map. Optics Laser Technol. 60, 111-115. doi: 10.1016/j.optlastec.2014.01.015

Mondal, B., and Mandal, T. (2017). A light weight secure image encryption scheme based on chaos, and DNA computing. J. King Saud Univ. Comput. Inform. Sci. 29, 499-504. doi: 10.1016/j.jksuci.2016.02.003

Priyanka, and Maheshkar, S. (2017). Region-based hybrid medical image watermarking for secure telemedicine applications. Multimedia Tools Appl. 76:36173647. doi: 10.1007/s11042-016-3913-1

Rehman, A., Liao, X. F., Hahsmi, M. A., and Haider, R. (2018). An efficient mixed inter-intra pixels substitution at 2bits-level for image encryption technique using DNA, and chaos. Optik 53, 117-134. doi: 10.1016/j.ijleo.2017.09.099 
Sun, S. (2018). A novel hyperchaotic image encryption scheme based on DNA encoding, pixel-level scrambling, and bit-level scrambling. IEEE Photon J. 10, 1-14. doi: 10.1109/JPHOT.2018.2817550

Wang, B., Xie, Y. J., Zhou, S. H., Zheng, X. D., and Zhou, C. J. (2018). Correcting errors in image encryption based on DNA coding. Molecules 23:1878. doi: $10.3390 /$ molecules 23081878

Wang, B., Zhang, Q., and Wei, X. P. (2020). Tabu variable neighborhood search for designing DNA barcodes. IEEE Trans. NanoBiosci. 19, 127-131. doi: 10.1109/ TNB.2019.2942036

Wang, X. Y., Wang, Y., Zhu, X. Q., and Luo, C. (2020). A novel chaotic algorithm for image encryption utilizing one-time pad based on pixel level, and DNA level. Optics Lasers Eng. 125, 105851. doi: 10.1016/j.optlaseng.2019.10 5851

Wang, Y., Lei, P., Yang, H. Q., and Cao, H. Y. (2015). Security analysis on a color image encryption based on DNA encoding, and chaos map. Comput. Electr. Eng. 46, 433-446. doi: 10.1016/j.compeleceng.2015.03.011

Wu, T. Y., Fan, X. N., Wang, K. H., Lai, C. F., Xiong, N., and Wu, J. M.T. (2019). A DNA computation based image encryption scheme for cloud CCTV systems. IEEE Access 181434-181443. doi: 10.1109/ACCESS.2019.294 6890

Wu, Y., Zhou, Y. C., Saveriades, G., Agaianc, S., Noonana, J. P., and Natarajan, P. (2013). Local Shannon entropy measure with statistical tests for image randomness. Inform. Sci. 222, 323-342. doi: 10.1016/j.ins.2012. 07.049

Xue, X. L., Zhang, Q., Wei, X. P., Guo, L., and Wang, Q. (2010a). A digital image encryption algorithm based on DNA sequence, and multi-chaotic maps. Neural Netw. World 20, 285-296.

Xue, X. L., Zhang, Q., Wei, X. P., Guo, L., and Wang, Q. (2010b). An image fusion encryption algorithm based on DNA sequence, and multichaotic maps. J. Comput. Theor. Nanosci. 7, 397-403. doi: 10.1166/jctn.2010. 1372

Yang, Y. G., Guan, B. W., Li, J., Li, D., Zhou, Y. H., and Shi, W. M. (2019). Image compression-encryption scheme based on fractional order hyperchaotics ystems combined with 2D compressed sensing, and DNA encoding. Optics Laser Technol. 119:105661. doi: 10.1016/j.optlastec.2019.105661

Zhang, J., Hou, D. Z., Ren, H. G., and Islam, N. (2016). Image encryption algorithm based on dynamic DNA coding, and Chen's hyperchaotic system. Math. Probl. Eng. 126, 1-11. doi: 10.1155/2016/6408741

Zhang, L. M., Sun, K. H., Liu, W. H., and He, S. B. (2017). A novel color image encryption scheme using fractional-order hyperchaotic system, and DNA sequence operations. Chin. Phys. B 26:100504. doi: 10.1145/3127404

Zhang, Q., Guo, L., and Wei, X. P. (2010). Image encryption using DNA addition combining with chaotic maps. Math. Comput. Model. 52, 2028-2035. doi: 10.1016/j.mcm.2010.06.005
Zhang, Q., and Wei, X. P. (2013). RGB color image encryption method based on Lorenz Chaotic system, and DNA computation. IETE Tech. Rev. 30, 404-409. doi: 10.4103/0256-4602.123123

Zhang, Q., Xue, X. L., and Wei, X. P. (2012). A novel image encryption algorithm based on DNA chain operation. Sci. World J. 2012:286741. doi: 10.1100/2012/ 286741

Zhang, S., and Gao, T. G. (2016). An image encryption scheme based on DNA coding, and permutation of hyper-image. Multimedia Tools Appl. 75, 1715714170. doi: 10.1007/s11042-015-2982-x

Zhang, X. C., Han, F., and Niu, Y. (2017). Chaotic image encryption algorithm based on bit Permutation, and dynamic DNA encoding. Hindawi Comput. Intell. Neurosci. 2017:6919675. doi: 10.1155/2017/6919675

Zhang, Y. Q., Wang, X. Y., Liu, J., and Chi, Z. L. (2016). An image encryption scheme based on the MLNCML system using DNA sequences. Optics Lasers Eng. 82, 95-103. doi: 10.1016/j.optlaseng.2016.02.002

Zhen, P., Zhao, G., Min, L. Q., and Jin, X. (2016). Chaos-based image encryption scheme combining DNA coding, and entropy. Multimedia Tools Appl. 75, 1-17. doi: 10.1007/s11042-015-2573-X

Zhou, S. H., Wang, B., Zheng, X. D., and Zhou, C. J. (2016). An image encryption scheme based on DNA computing, and cellular automata. Discr. Dyn. Nat. Soc. 2016:5408529. doi: 10.1155/2016/5408529

Zhou, Y., Hua, Z., Pun, C. M., and Chen, C. L. P. (2015). Cascade chaotic system with applications. IEEE Trans. Cybern. 45, 2001-2012. doi: 10.1109/TCYB. 2014.2363168

Zhu, E. Q., Chen, C. Z., Rao, Y. S., and Xiong, W. C. (2020). Biochemical logic circuits based on DNA combinatorial displacement. IEEE Access 8, 3409634103. doi: 10.1109/ACCESS.2020.2974024

Zhu, S. Q., Li, J. Q., and Wang, W. H. (2017). Security analysis of improved image encryption method based on DNA coding, and chaotic map. Appl. Res. Comput. 34, 3090-3093.

Zhu, W., Yang, G., Chen, L., and Chen, Z. Y. (2014). An improved image encryption algorithm based on double random phase encoding, and chaos. Acta Opt. Sin. 34:0607001. doi: 10.3788/AOS201434.0607001

Conflict of Interest: The authors declare that the research was conducted in the absence of any commercial or financial relationships that could be construed as a potential conflict of interest.

Copyright $\odot 2021$ Xue, Jin, Zhou and Zhou. This is an open-access article distributed under the terms of the Creative Commons Attribution License (CC BY). The use, distribution or reproduction in other forums is permitted, provided the original author(s) and the copyright owner(s) are credited and that the original publication in this journal is cited, in accordance with accepted academic practice. No use, distribution or reproduction is permitted which does not comply with these terms. 